ARTICLE

https://doi.org/10.1038/s41467-020-15714-x

\title{
OPEN
}

\section{Lgr5+ telocytes are a signaling source at the intestinal villus tip}

Keren Bahar Halpern', Hassan Massalha', Rachel K. Zwick², Andreas E. Moor (D) ${ }^{3}$, David Castillo-Azofeifa (D) ${ }^{2}$,

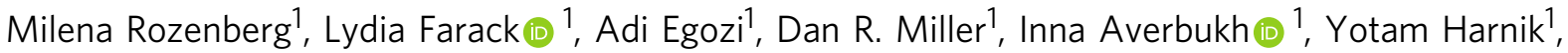
Noa Weinberg-Corem ${ }^{4}$, Frederic J. de Sauvage ${ }^{5}$, Ido Amit (i) ${ }^{6}$, Ophir D. Klein (1) ${ }^{2,7}$, Michal Shoshkes-Carmel ${ }^{4 凶} \&$ Shalev Itzkovitz ${ }^{1 凶}$

The intestinal epithelium is a structured organ composed of crypts harboring Lgr5+ stem cells, and villi harboring differentiated cells. Spatial transcriptomics have demonstrated profound zonation of epithelial gene expression along the villus axis, but the mechanisms shaping this spatial variability are unknown. Here, we combine laser capture micro-dissection and single cell RNA sequencing to uncover spatially zonated populations of mesenchymal cells along the crypt-villus axis. These include villus tip telocytes (VTTs) that express Lgr5, a gene previously considered a specific crypt epithelial stem cell marker. VTTs are elongated cells that line the villus tip epithelium and signal through Bmp morphogens and the noncanonical Wnt5a ligand. Their ablation is associated with perturbed zonation of enterocyte genes induced at the villus tip. Our study provides a spatially-resolved cell atlas of the small intestinal stroma and exposes Lgr5+ villus tip telocytes as regulators of the epithelial spatial expression programs along the villus axis.

\footnotetext{
${ }^{1}$ Department of Molecular Cell Biology, Weizmann Institute of Science, 7610001 Rehovot, Israel. ${ }^{2}$ Program in Craniofacial Biology and Department of Orofacial Sciences, University of California, San Francisco, CA 94110, USA. ${ }^{3}$ Institute of Molecular Cancer Research, University of Zurich, 8002 Zurich, Switzerland. ${ }^{4}$ Department of Developmental Biology and Cancer Research, The Institute for Medical Research Israel-Canada, The Hebrew University Hadassah Medical School, 9112001 Jerusalem, Israel. ${ }^{5}$ Department of Molecular Oncology, Genentech, San Francisco, CA 94080, USA. ${ }^{6}$ Department of Immunology, Weizmann Institute of Science, 7610001 Rehovot, Israel. ${ }^{7}$ Department of Pediatrics and Institute for Human Genetics, University of California, San Francisco, CA 94110, USA. ${ }^{凶}$ email: michal.shoshkes@mail.huji.ac.il; shalev.itzkovitz@weizmann.ac.il
} 
$\mathrm{T}$ he small intestine is a structured organ composed of repeating crypt-villus units. $L g r 5+$ epithelial stem cells at the base of the crypts continuously divide to give rise to proliferative progenitors that migrate towards the villi ${ }^{1,2}$. As they approach the crypt exits, these progenitors become post-mitotic and differentiate into distinct lineages-absorptive enterocytes, mucussecreting goblet cells, enteroendocrine cells and tuft cells. The differentiated cells operate for about three days as they continue to migrate along the villus walls until they are shed from the villi tips ${ }^{3}$.

Villi cells have traditionally been considered 'terminally-differentiated' in the sense that they have irreversibly committed to a functional cell state, which they carry from birth to death. Recent studies challenged this view and demonstrated that villi epithelial cells constantly change their functional state, such that around $85 \%$ of enterocyte genes are expressed in a non-uniform manner along the villus axis ${ }^{4}$. Enterocytes first implement anti-microbial programs at the base of the villi, then shift to sequential absorption of amino acids, carbohydrates and lipids in distinct villi zones, finally upregulating genes associated with cell adhesion and immune modulation at the villi tips. Similarly, enteroendocrine cells change the types of hormones they produce as a function of their position along the villus axis 5,6 . The epithelial lineages along the intestinal villus therefore exhibit profound spatial heterogeneity.

What are the mechanisms that facilitate the spatial heterogeneity of the villus epithelium? One mechanism could be an internal 'clock', whereby epithelial cells are pre-programmed to turn distinct functions on and off at different times. A second mechanism could be a zonated response to spatial gradients of nutrients and bacteria at the luminal sides of the tissue. Yet a third mechanism could entail zonated molecular cues that originate in the lamina propria, the underlying stroma at the basal sides of the epithelial layer ${ }^{7-11}$. Here, we use spatially-resolved transcriptomics to characterize the zonated stromal gene expression signatures along the crypt-villus axis. We identify four mesenchymal cell populations residing at distinct crypt-villus zones. These include a sub-population of telocytes localized at the villus tip that is marked by Lgr5, a gene previously considere a specific marker of epithelial crypt stem cells. We suggest that Lgr5+ villus tip telocytes may regulate the epithelial gene expression programs at the villus tip.

\section{Results}

A spatial expression atlas of the small intestinal stroma. To obtain a global view of spatial heterogeneity of stromal gene expression we used Laser Capture Microdissection (LCM) to isolate four stromal zones along the jejunum crypt-villus axis from five mice (Fig. 1a, b). We performed RNA sequencing of these segments, yielding a coarse zonation map (Supplementary data 1). We focused on the zonation patterns of ligands and receptors ${ }^{12,13}$, since these are likely to implement zonated crosstalk with the epithelium (Fig. 1c, Supplementary Fig. 1a, b, Supplementary data 2). Examples of ligand/receptor genes that exhibited higher expression in the crypt stroma included Grem1, encoding the BMP pathway inhibitor Gremlin $1^{14}$ (Fig. 1c) and Il18r1, the receptor for Il18. The cytokine Ill8 has been shown to be expressed in enterocytes at the lower villi zones, as part of an anti-microbial gene module 4 . Using single molecule Fluorescence in-situ Hybridizatoin $\left(\mathrm{smFISH}^{15}\right)$, we identified type 3 innate lymphoid cells (ILC3) expressing Il18r1 at the stromal side of these zones (Supplementary Fig. 1c-g), suggesting spatial recruitment of these immune cell components by zonated enterocyte Il18 signal.

Lgr5 is abundantly expressed in villus tip stromal cells. We next focused on stromal ligands and receptors that were zonated towards the villi tips in our stromal LCM-RNAseq data. These included Bmp4, a morphogen that was shown to inhibit epithelial proliferation ${ }^{16}$ and to control enteroendocrine cell differentiation pathways at the tip ${ }^{5}$ (Fig. 1c). Other villus tip molecules included the non-canonical Wnt ligand Wnt4 and the epidermal growth factor Egf. Egfr, the receptor for Egf, has elevated expression levels in villus tip enterocytes ${ }^{4}$, constituting another example of correlated spatial expression of pairs of epithelial and stromal ligands and their matching receptors (Supplementary data 2). We further analyzed zonated proteins constituting the extracellular matrix (ECM, also termed the 'matrisome'17), identifying distinct collagens and matrix metalloproteinases that are differentially zonated between the crypt and villus stroma (Supplementary Fig. $1 \mathrm{~h}$ ). These included the crypt enriched Lama2, encoding the laminin subunit alpha- 2 and the villus enriched Lama5, encoding the laminin subunit alpha-5, previously shown to be differentially zonated at the protein level ${ }^{18,19}$.

Our zonation analysis revealed Lgr5 to be one of the most highly expressed receptors in the villus tip stroma (Fig. 1c, Supplementary data 1). Lgr5 has been shown to be a specific marker of epithelial stem cells at the crypt base ${ }^{2,15}$. It was therefore unexpected to observe elevated expression levels of this gene at the lamina propria of the villus tip. To validate this finding, we performed smFISH and detected abundant localized expression of Lgr5 transcripts in PDGFRa+ telocytes that coexpressed Bmp4, a classic villus tip ligand ${ }^{20}$ (Fig. 2a-e). Telocytes are large mesenchymal cells with elongated extensions that stain positively for the PDGFRa surface marker. They form intricate contacts with both epithelial cells and other stromal cell types ${ }^{9,21}$. Telocytes that surround the crypts constitute important niche cells that secrete canonical Wnt morphogens and Rspo3 to maintain stemness of crypt Lgr5+ epithelial stem cells $1,11,22,23$. The molecular identities of telocytes residing in the villi stroma have thus far not been characterized.

The expression levels of Lgr5 in the villus tip telocytes (VTTs) were comparable to the expression levels in the epithelial crypt base columnar stem cells (mean expression of $0.109 \pm 0.012$ $\mathrm{mRNAs} / \mu \mathrm{m}^{-3}$ in tip telocytes vs. $0.104 \pm 0.008 \mathrm{mRNAs} / \mu \mathrm{m}^{-3}$ in crypt stem cells, (Fig. 2f). VTTs expressed both Lgr5 and its ligand $R_{s p o} 3^{24}$ (Fig. 2g). Notably, mRNAs of Rspo3 were localized along telopodes- thin PDGFRa + extensions of the telocytes that extended towards the lamina propria and away from the cell body, where Lgr5 mRNAs were localized (Fig. 2g).

Several mouse models have been developed to investigate intestinal Lgr5 + crypt epithelial stem cells. These include models for ablating $L g r 5$ cells $^{25}$ and for tracing their progenies ${ }^{2}$. We asked whether the knock-in constructs in these mice were also expressed in Lgr5+ VTTs. Indeed, VTTs were clearly seen in Lgr5-GFP-DTR mice ${ }^{25}$ (Fig. 3a, b) and Lgr5-EGFP-Ires-CreERT2 mice $^{2}$ (Fig. 3c, d). All GFP+ stromal cells had Lgr5 mRNA in the Lgr5-GFP-DTR mice (60 out of 60 cells counted over 10 villi from 2 mice) and no Lgr5+ cells at the villus tip stroma lacked GFP. The proportions of $\operatorname{Lgr} 5+$ VTTs positive for the EGFP knock-in in the Lgr5-EGFP-Ires-CreERT2 mice were lower than the proportion of EGFP+ crypts ( $13 \% \pm 3 \%$ vs. $35 \% \pm 6 \%$, counted over 22 villi in two mice), indicating a partial silencing of this knock-in reporter in the mesenchymal lineage in this mouse model. In summary, Lgr5 specifically labels telocytes localized at the villus tip, in addition to its documented role as a marker of epithelial crypt stem cells.

Single cell transcriptomics of villus mesenchymal cells. We next sought to obtain the global gene expression signatures of Lgr5+ VTTs and of other intestinal mesenchymal cell populations. LCMseq provides a spatial map of gene expression along the 
a

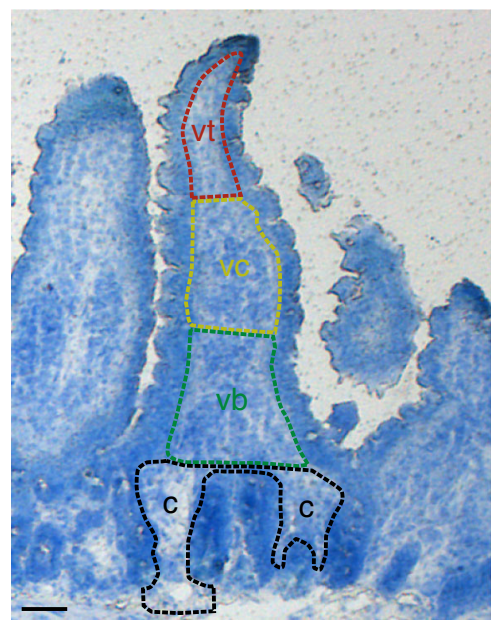

b

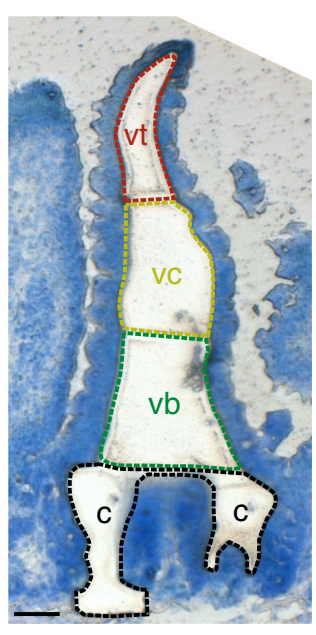

C

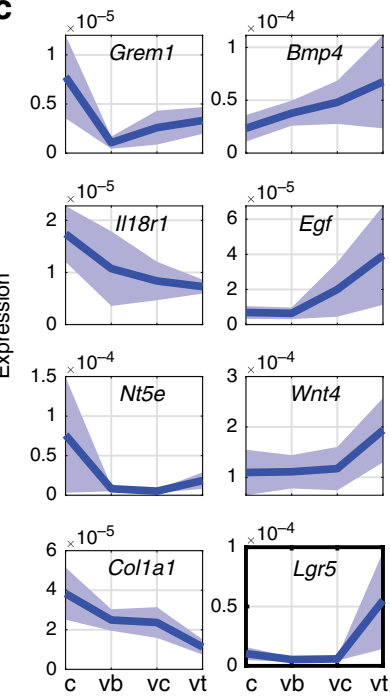

Fig. 1 Spatial transcriptomics of the intestinal stroma. a-b LCM of four zones along the crypt-villus axis before (a) and after (b) laser dissection. c-crypt, vb-villus bottom, vc-villus center, vt-villus tip. Scale bar-50 $\mu \mathrm{m}$. c Representative spatial LCMseq expression profiles of stromal ligands and receptors zonated towards the crypt (left) or villus tip (right). Units are fraction of sample mRNA, patches are SEM.

crypt villus axis, however each zone represents a mixture of diverse cell types, including mesenchymal cells, endothelial cells, immune cells ${ }^{26}$ and enteric neurons ${ }^{27}$. Unraveling the cellular origins of the zonated transcripts requires single cell transcriptomics. Extracting telocytes is challenging due to their long thin extensions and their entrenchment within the ECM. PDGFRa was previously shown to be expressed in telocytes throughout the crypt-villus axis ${ }^{9}$. We therefore used fluorescence activated cell sorting to enrich for PDGFRa + cells that included the intestinal telocytes, as well as other mesenchymal cell types (Supplementary Fig. 2). We performed single cell RNA sequencing on these cells using the MARS-seq protocol ${ }^{28}$. Although we enriched for PDGFRa + cells, our scRNAseq map included predominantly epithelial cells and immune cells, most likely obtained due to the attachment of PDGFRa + telopode fragments during the dissociation processes. Importantly, our extraction also yielded 329 pure mesenchymal cells (Fig. 4a-c, Methods).

We focused on the mesenchymal cell clusters that were positive for PDGFRa. Reclustering these 329 cells yielded four distinct mesenchymal cell populations (Fig. 4d-e, Supplementary data 3). We used Seurat ${ }^{29}$ to identify distinct gene expression markers for each of the four cell populations (Fig. 4e, Supplementary Fig. 3, Supplementary data 4). To identify potential zonation of these mesenchymal cell types we used our LCMseq map (Supplementary data 1) to examine the expression of the marker genes from each cluster (Fig. 4f, Methods). Using the marker gene identities and their spatial patterns, we annotated the clusters as crypt telocytes, enteric mesothelial fibroblasts, myofibroblasts and villus tip telocytes (VTTs). 10 cells out of the 329 mesenchymal cells were $\operatorname{Lgr} 5+, 7$ of which were in the VTT cell population (Fig. 4e, hypergeometric $p=0.0121$ ). The remaining 3 Lgr $5+$ cells in our scRNAseq data were interspersed with 1 cell in each of the remaining three cell populations.

We used smFISH to validate the spatial patterns of expression of the cell type markers revealed by the scRNAseq measurements. Grem1 and Sfrp1 expression was elevated in telocytes at the bottom of the crypts (Supplementary Fig. 4a, c). Ly6a and Angptl4 expression was elevated in VTTs (Supplementary Fig. 4b, c). Notably, although the scRNAseq data suggested higher levels of Bmp2 and Bmp4 ligands in crypt telocytes, our smFISH measurements showed significantly higher levels of Bmp2 and
Bmp4 in VTTs, in line with the LCM measurements (Fig. 1c, Supplementary Fig. 4c). This inconsistency might stem from the low capture rate of VTT transcripts. Enteric mesothelial fibroblasts ${ }^{27}$ were marked by Thbs2, Fibin and Rgs5, as well as Angptl2, a gene previously shown to inhibit $\mathrm{Bmp}^{30}$. These were interspersed throughout the crypt-villus axis and were localized at the core of the villus, away from the epithelial layer (Supplementary Fig. 4d), as were myofibroblasts, marked by Acta2, Tagln and Aldh1a1 (Fig. 4e).

VTTs, as well as crypt telocytes, expressed high levels of ECM components such as Col3a1, Colla1, and Timp2 (Supplementary data 3). VTTs expressed elevated levels of genes encoding microfibrillar proteins such as Mfap5, Emilin2, and Fbn1 (Fig. 5a, Supplementary Fig. 3, Supplementary data 3). Differential gene expression between crypt telocytes and VTTs further revealed elevated levels of the non-canonical Wnt ligand Wnt5a in VTTs (Fig. 5a,b), suggesting that VTTs implement a switch from canonical to non-canonical Wnt signaling ${ }^{31,32}$. Indeed, we observed broad expression of Axin2, a transcriptional target of canonical Wnt signaling, along the villi epithelial cells, with a decrease at the villus tip (Fig. $5 \mathrm{c}$ ), the zone of stromal Wnt5a expression.

VTT ablation perturbs villus tip epithelial gene expression. Enterocytes undergo profound gene expression changes as they approach the villus tip, a few hours before they are shed off into the lumen ${ }^{4}$. The villus tip enterocyte expression program includes induction of the adherens junction protein E-cadherin (Fig. 5d), the AP-1 transcription factor components Fos (Fig. 5c) and Jun, the transcription factor Klf4 and the immune-modulatory purinemetabolism program consisting of Ada, Nt5e, and Slc28a2 $2^{4}$. To assess the role of VTTs in regulating enterocyte zonation at the tip, we ablated Lgr5 cells using the Lgr5-GFP-DTR mouse model $^{25}$, in which Lgr5+ cells express diphtheria toxin (DT) receptor and GFP. In this mouse model, $100 \%$ of Lgr5+ VTTs were positive for GFP (60 out of 60 cells counted over 10 villi from 2 mice, Fig. 3a, b, Fig. 6a, Supplementary Fig. 5a). 24 h after DT administration, $L g r 5+$ VTTs were completely ablated, as were the crypt Lgr5+ stem cells (Fig. 6b). As previously shown, Lgr5 expression re-appeared in the crypt bottom $48 \mathrm{~h}$ after ablation ${ }^{25}$, 
a

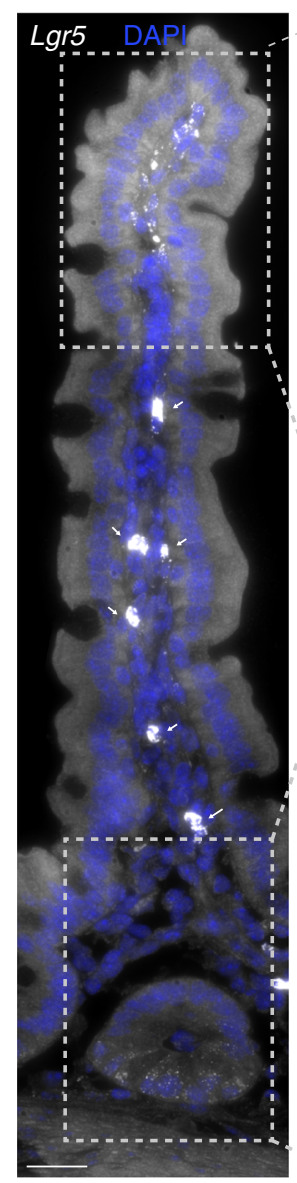

b

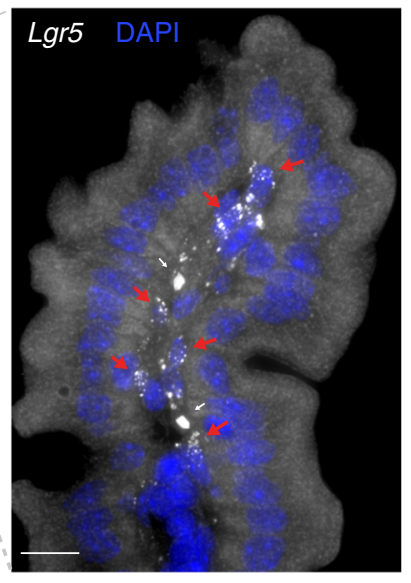

C

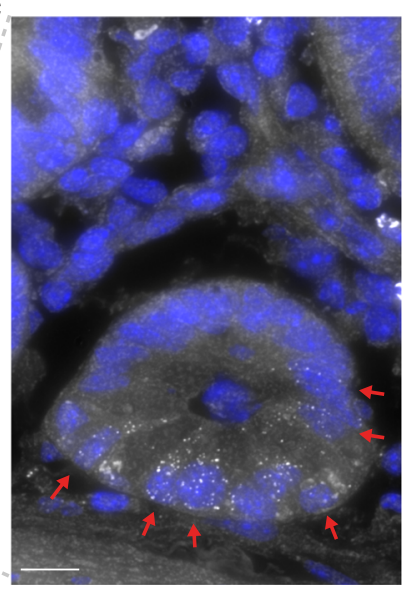

d

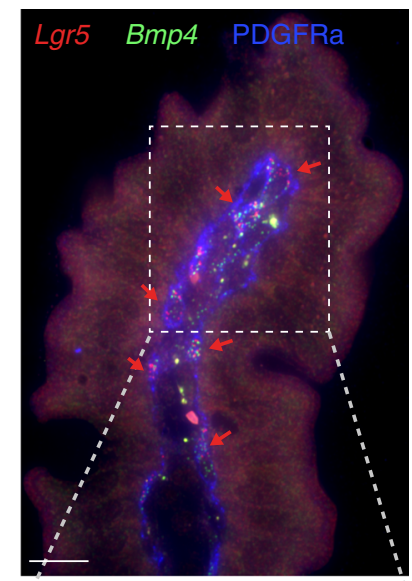

e

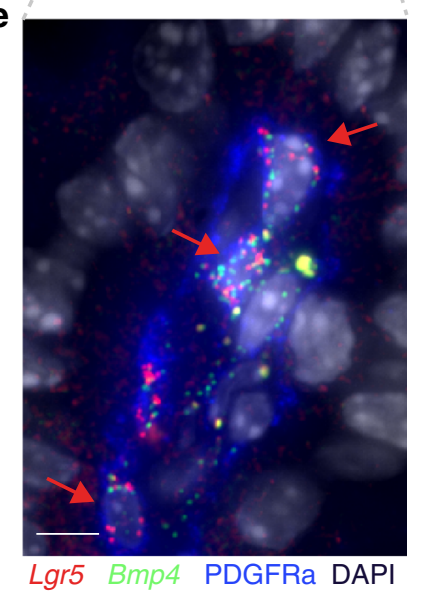

$\mathbf{f}$

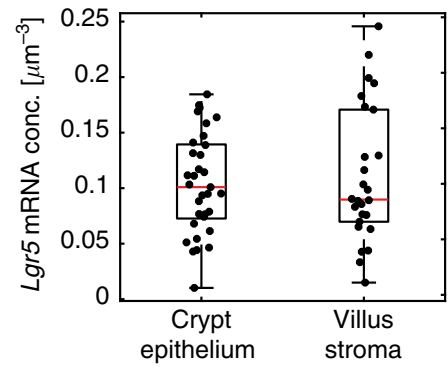

$\mathbf{g}_{\text {Lgr5 }}$ Rspo3 PDGFRa
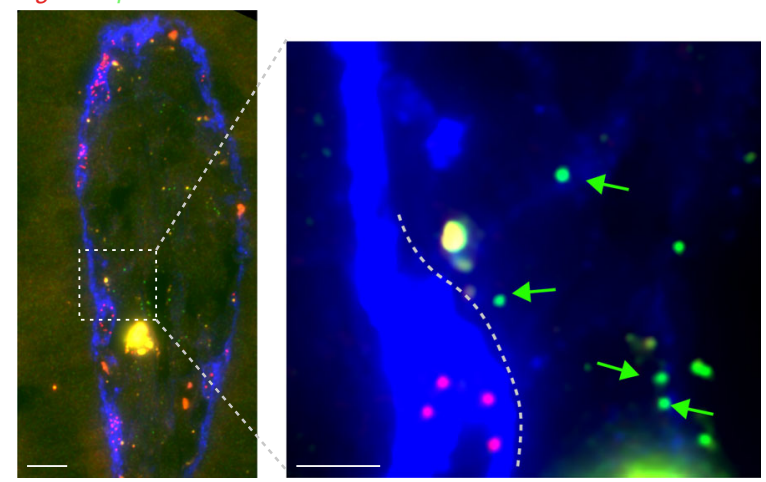

Fig. 2 Lgr5 is expressed abundantly in villus tip telocytes. a smFISH of Lgr5, DAPI in blue, Scale bar-20 $\mu$ m. b Blow up of villus tip, Scale bar-10 $\mu$ m. In a, b thin white arrows point at autofluorescent blobs. c blow up of crypt, Scale bar-10 $\mu \mathrm{m}$. Red arrows in b-c point to Lgr5 positive cells. $\mathbf{d}$ Lgr5 mRNA (red dots) expressed in PDGFRa + VTTs that co-express Bmp4 mRNA (green dots). Scale bar-10 $\mu \mathrm{m}$. Red arrows point to Lgr5 and Bmp 4 double positive cells. e Blow up of the region boxed in $\mathbf{d}$. Scale bar $-5 \mu \mathrm{m}$. $\mathbf{f} \operatorname{Lgr} 5 \mathrm{mRNA}$ concentrations in VTTs are comparable to those in $\operatorname{Lgr} 5+$ crypt base columnar cells $(n=25$ cells examined over 2 mice for each region). Boxes show 25-75 percentiles of the smFISH expression, horizontal red lines are medians. Whiskers, extend to the most extreme data point within 1.5× the interquartile range (IQR) from the box; $\mathbf{g}$ ) Rspo3 mRNAs are localized on telopodes that extend away from the cell bodies of the VTTs. VTTs are marked by Lgr5 mRNA (red dots), Rspo3 mRNA (green dots) is localized away from the cell body, PDGFRa antibody mark VTTs cell bodies and telopodes. Scale bar-10 $\mu \mathrm{m}$, in inset, green arrows point to Rspo3 mRNAs (green dots) localized on PDGFRa telopodes (blue).

Telocyte cell body is marked by white dashed line. inset Scale bar- $5 \mu \mathrm{m}$.

yet not at the villus tip, as evident by the complete loss of GFP signal (Fig. 6c) and Lgr5 mRNA (Supplementary Fig. 5).

Ablation of Lgr5+ crypt base columnar cells has been shown to initiate a regenerative program in the remaining crypt cells ${ }^{33}$. Importantly, migration of epithelial cells from the crypt to the villus tip takes $3-5$ days ${ }^{1,3}$. We therefore argued that at short times following ablation of Lgr5+ cells, reduction in the expression of genes normally confined to the villus tip epithelium would be a result of loss of signals from the Lgr5+ VTTs, rather than incoming enterocytes, the state of which was perturbed in the crypt. To obtain a global view of the impact of VTTs on villus tip enterocyte gene expression, we therefore performed RNA 
a

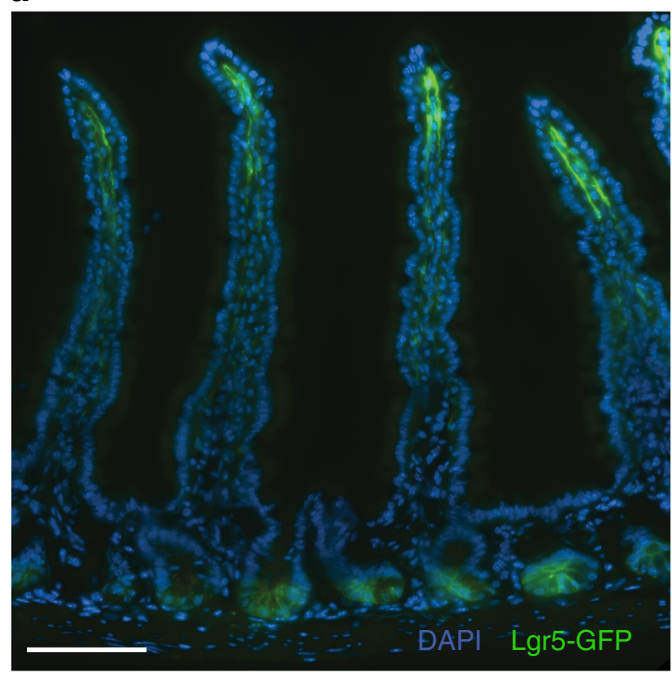

b

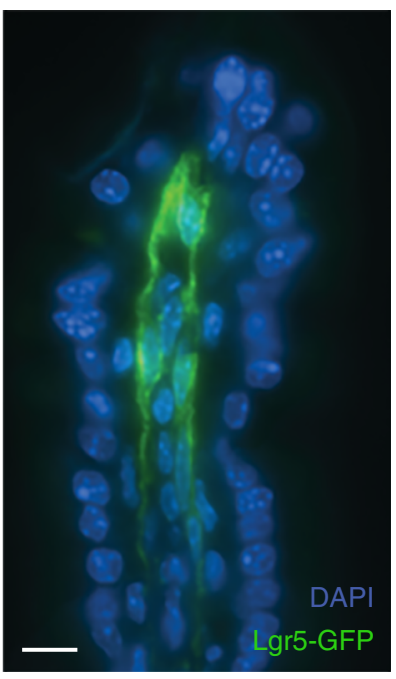

C

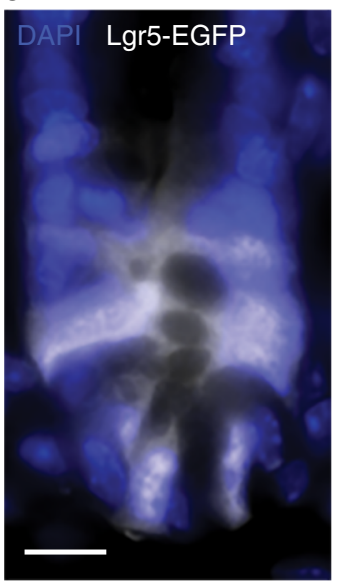

d

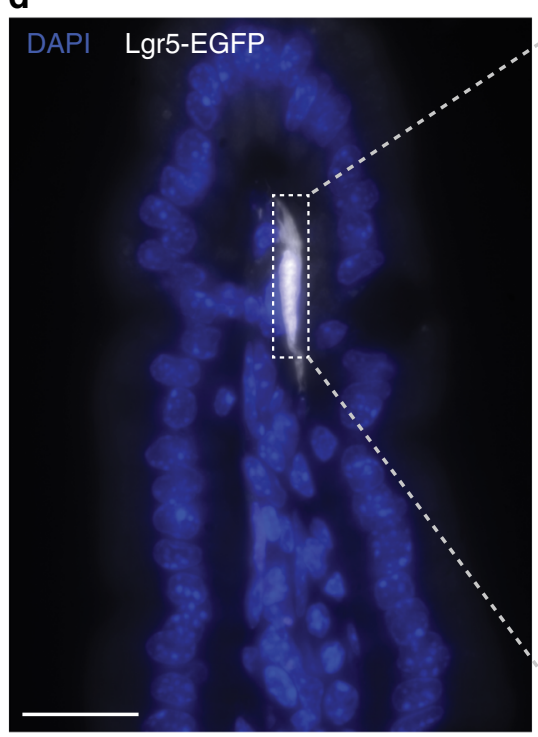

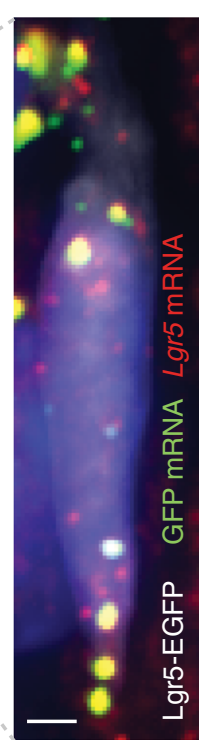

Fig. 3 Lgr5 expression at the villus tip of Lgr5-knock-in mouse models. a GFP fluorescence (green) observed in both crypt base columnar cells (CBCs) and VTTs in Lgr5-GFP-DTR mice. Scale bar- $100 \mu \mathrm{m}$. b blow up of villus tip with GFP+VTTs, scale bar-10 $\mu \mathrm{m}$. c EGFP fluorescence of crypt base columnar cells in Lgr5-EGFP-IRES-creERT2 mice. Scale bar-10 $\mu \mathrm{m}$. d EGFP fluorescence in VTTs. The Lgr5-EGFP knock-in construct is expressed in a patchy manner in the villus tip stroma. Scale bar-20 $\mu \mathrm{m}$. Blow up shows Lgr5 mRNAs in red, EGFP mRNA in green and DAPI in blue. Scale bar- $2 \mu \mathrm{m}$. Yellow blobs are autofluorescent elements.

sequencing of the epithelial layer $48 \mathrm{~h}$ after DT administration (Supplementary data 5, Fig. 6d, e, Methods). We identified a significant repression in enterocyte genes that were normally zonated towards the tip (Spearman correlation of $R=-0.53, p<$ $10^{-60} 48 \mathrm{~h}$ post ablation between expression change following ablation and expression zone, Fig. 6e, Supplementary data 6). Villus tip enterocyte genes that were strongly reduced in expression upon VTT ablation included the purine metabolism module genes Slc28a2, Nt5e, and Ada (Fig. 6d-i).

Additional prominent villus tip enterocyte genes include the transcription factors $K l f 4$ and Fos, Cdh1, encoding E-cadherin and $E g f r^{4}$. These genes have a relatively high basal expression level either throughout the villi axis (Cdh1, Klf4, and Fos) or at the crypt (Egfr). Expression changes in enterocyte at the villus tip could thus be masked for such genes in bulk RNA sequencing (Fig. 6d). To assess their expression changes, we therefore performed smFISH and measured expression specifically at the tip enterocytes, identifying a significant reduction of Egfr, Cdh1,
Klf4, and Fos, in addition to Ada, Nt5e and Slc28a2 (Fig. 6f-i). Creb3l3, an enterocyte gene that is elevated at the villi tip, did not exhibit changes in expression upon VTT ablation. Epithelial Axin2 expression did not change following VTT ablation (Supplementary data 5). These results demonstrate that VTTs are important regulators of the spatial expression programs of enterocytes at the villus tip, instructing the epithelial expression of key genes such as Egfr, Cdh1, Klf4, Fos, Nt5e, Ada, and Slc28a2.

To assess the long-term consequences of VTT ablation we examined small intestinal tissue three weeks after VTT ablation. At this time point, VTTs re-appeared in $65 \%$ of the villi tips (Fig. 7, $46 \mathrm{GFP}+$ villi out of 71 villi, counted over 2 mice). Notably, the mRNA levels of the enterocyte villus tip genes $A d a$, Nt5e, Egfr, Fos, and Klf4 remained significantly lower in the villi tips that lacked VTTs, compared to tips where VTTs reappeared (Fig. 7, mRNA conc. more than 2-fold higher in GFP+ vs. GFPvilli). In contrast, $C d h 1$ and Creb3l3 exhibited only a slight, yet statistically significant higher expression in the GFP+ villi (1.2 
a
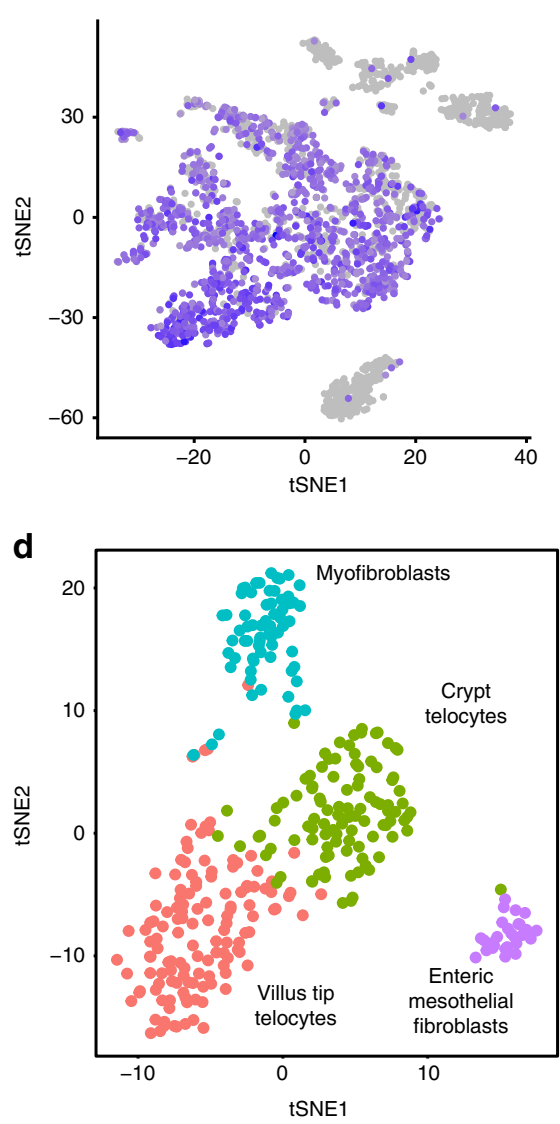

$\mathbf{f}$

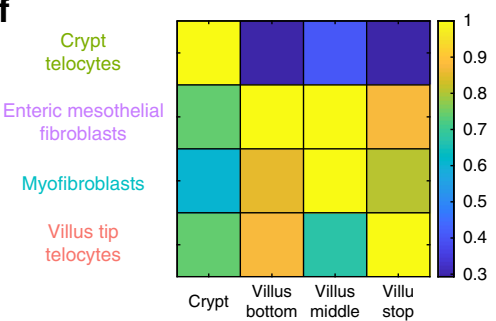

b

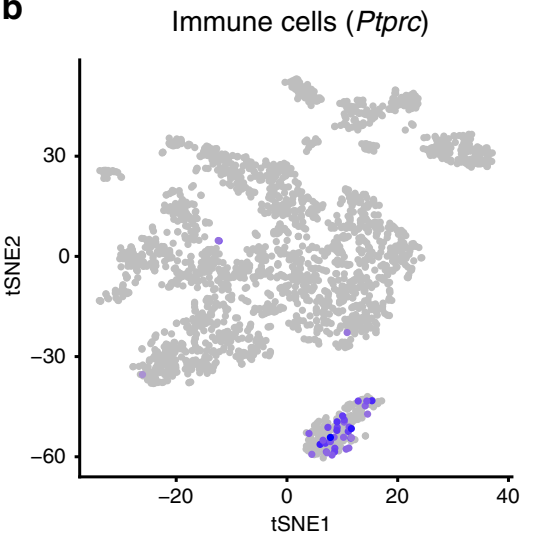

$\mathbf{e}$
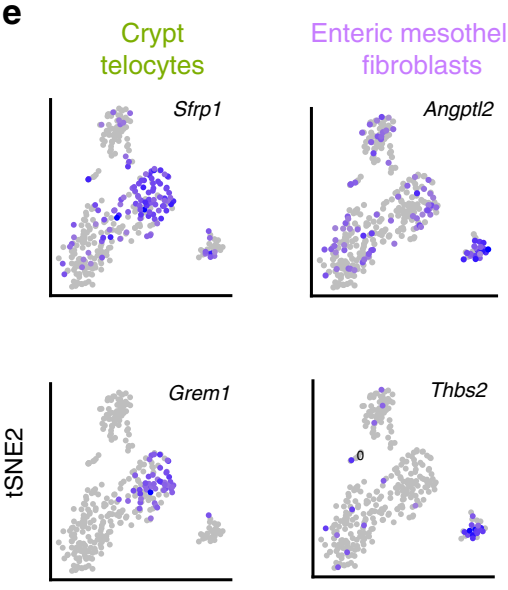

Enteric mesothelia fibroblasts
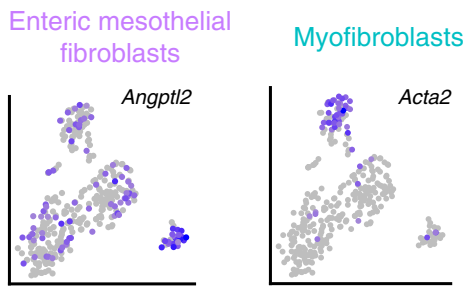

Fibroblasts (Pdgfra)
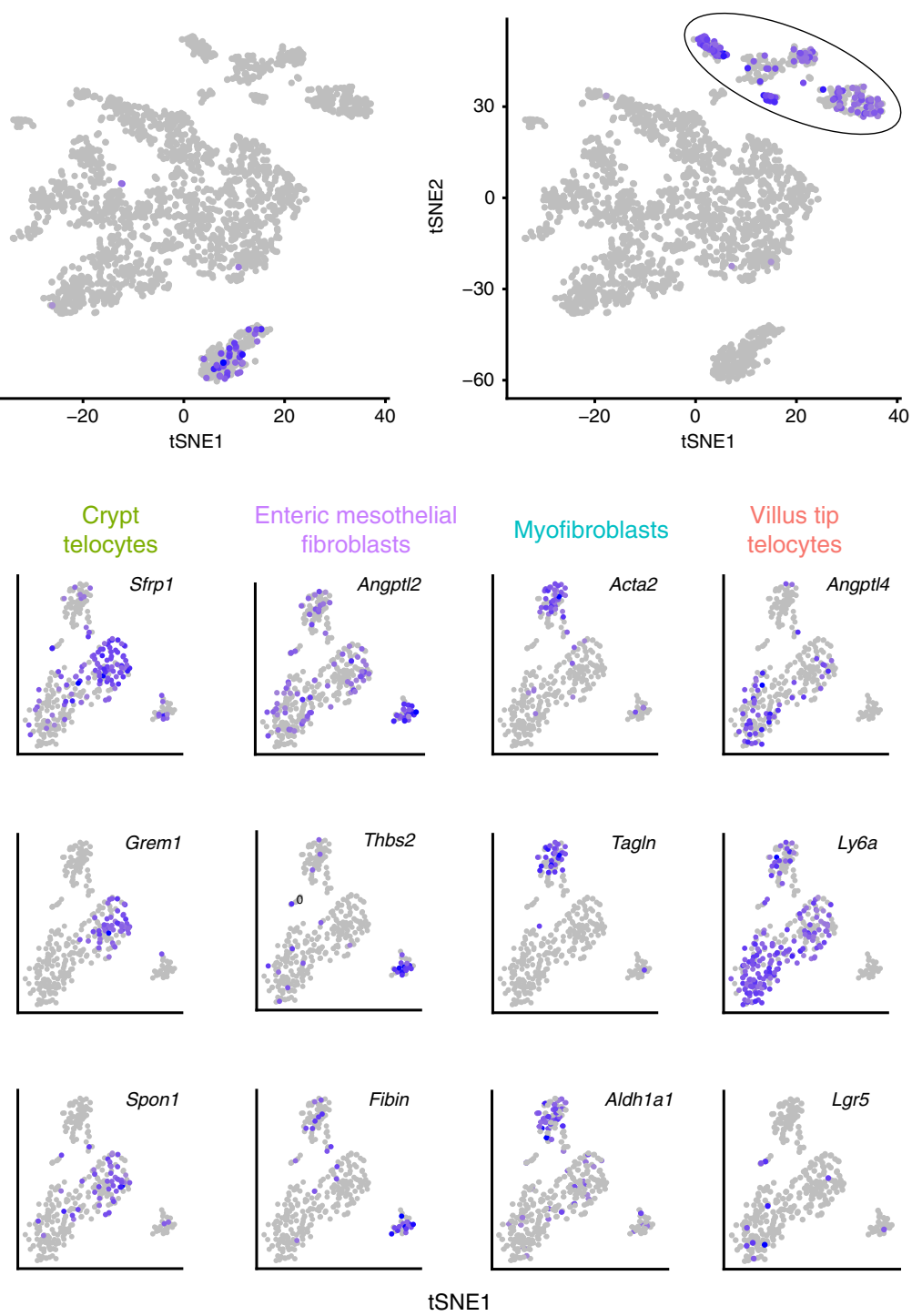

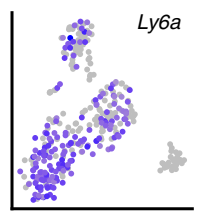

Villus tip telocytes
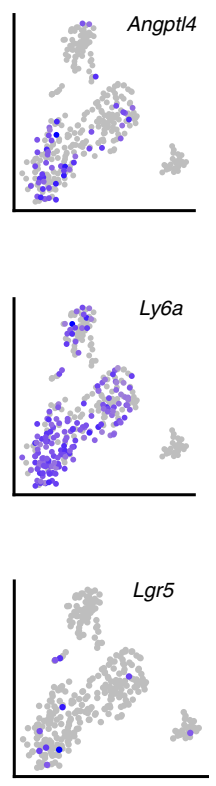

Fig. 4 Single cell transcriptomics of the intestinal stroma identifies four spatially-stratified mesenchymal cell populations. a-c tSNE plots of sequenced single cells highlighting the expression of Epcam, an epithelial marker (a), Ptprc, encoding CD45, a pan-immune marker (b) and Pdgfra (c) a mesenchymal marker. Purple hue indicates log expression levels. $\mathbf{d}$ tSNE plot of the Pdgfra+ cells from the cluster circled in c. Colors denote the identified four clusters, obtained by re-clustering the Pdgfra+ cluster cells in c. e tSNE plots colored by selected markers for the four mesenchymal cell clusters (see Supplementary Fig. 3 and Supplementary data 4 for the complete list of markers). Lgr5 is significantly enriched in the Villus Tip Telocytes ( 7 out of 10 Lgr5+ cells belong to the VTT cluster, hypergeometric $p=0.0121$ ). f Summed expression of the top markers for each mesenchymal cell cluster in the LCMseq data, normalized by the maximum across zones (Methods).

fold and 1.13 fold for Cdh1 and Creb3l3, respectively, in GFP+ vs. GFP-). The correlation between VTT re-appearance and villus tip epithelial gene expression at this time point, when crypts have already returned to normal homeostatic state, further supports the instructive role of VTTs in regulating the enterocyte villus tip expression program.

\section{Discussion}

Our study exposed the spatial diversity of mesenchymal cells along the crypt-villus axis. We identified a cell population of Lgr5+ VTTs that form a highly localized niche for the villus tip epithelium, facilitating the massive transcriptional changes that enterocytes undergo before they are shed from the tissue ${ }^{4}$. VTTs are a source of Bmp ligands (Fig. 2d, e, Supplementary Fig. 4c,
Supplementary data 1) and may implement a switch from canonical to non-canonical Wnt signaling, through the expression of the non-canonical Wnt ligand Wnt5a (Fig. 5b). Similar spatially antagonistic expression of stromal Wnt5a expression and canonical Wnt activity has been recently demonstrated in the mouse prostate ${ }^{31}$. This switch may be important for the induction of E-cadherin, a major component of the adherens junction that is strongly induced at the villus tip enterocytes (Fig. 5d) ${ }^{34}$. Wnt5a is also an activator of the AP1 transcription factors ${ }^{35}$, composed of the enterocyte villus tip genes Fos and Jun ${ }^{4}$. VTTs also express components of the ECM, such as microfibrillar proteins (Fig. 5a), and therefore their ablation could result in perturbed ECM composition, indirectly affecting enterocyte gene expression. Future work, involving VTT-specific ablation of signaling 
a

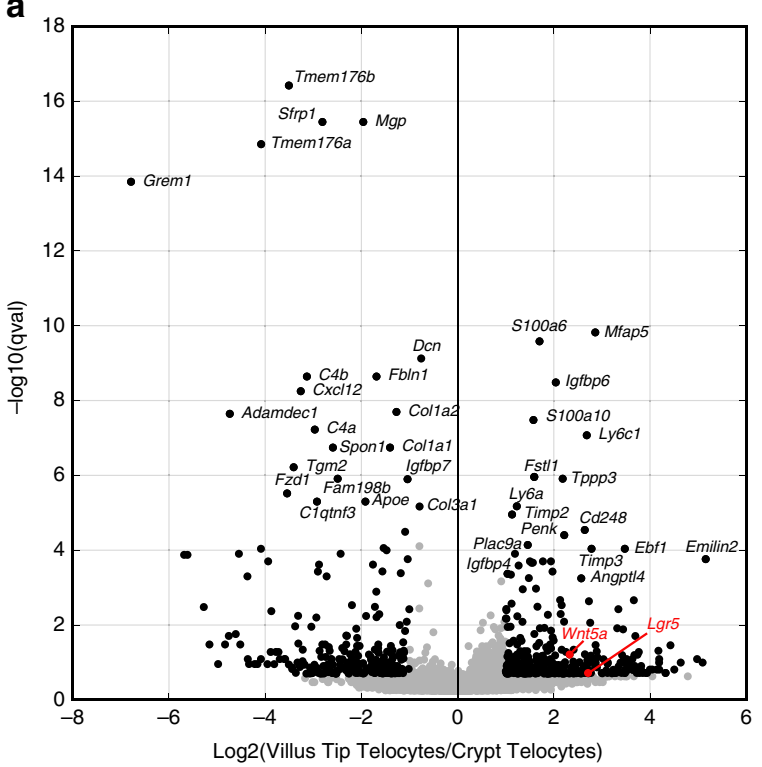

b

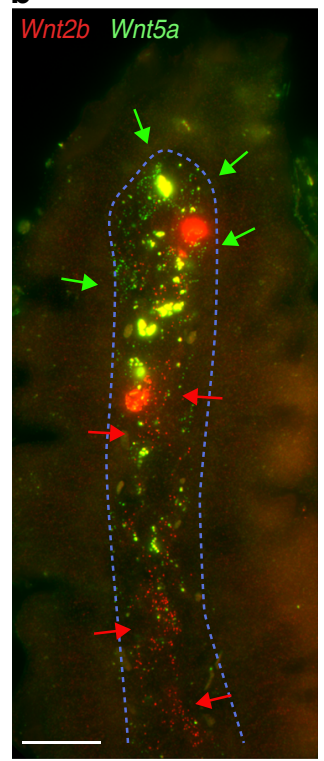

c

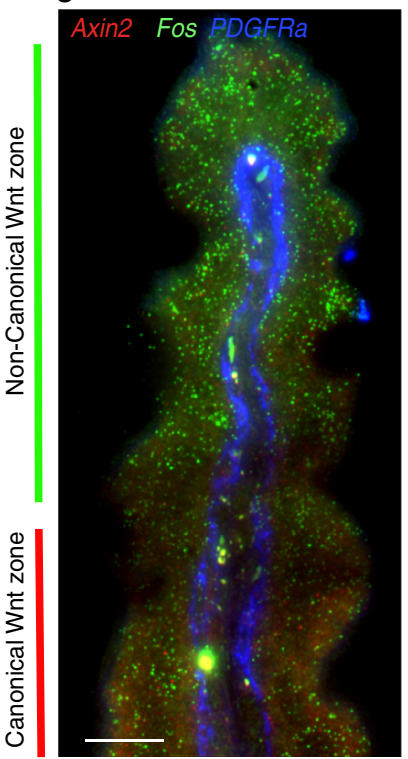

d

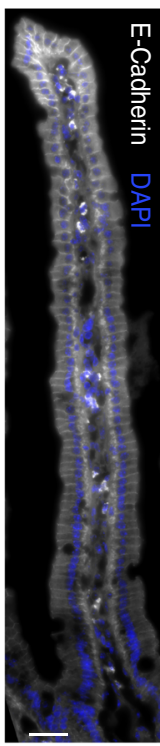

Fig. 5 VTTs implement a spatial switch from canonical to non-canonical Wnt signaling. a Volcano plot demonstrating differential gene expression

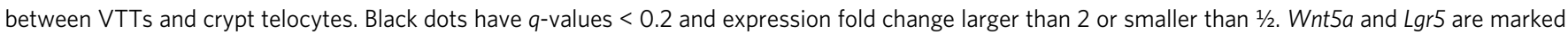
in red. b Spatial shift in the stroma from the expression of canonical Wnt2b (red dots, marked by red arrows) to non-canonical Wnt5a (green dots, marked by green arrows). Scale bar-20 $\mu \mathrm{m}$. c Axin2 (red dots) is expressed broadly along the villus axis and repressed at the villus tip. Fos (green dots) is highly expressed in villus tip enterocytes. scale bar-20 $\mu \mathrm{m}$. d E-Cadherin protein (gray), encoded by Cdh1 gene, is induced in villus tip enterocytes. Large blobs in b-d are autofluorescent signals originating in immune cells. scale bar-30 $\mu \mathrm{m}$.

components such as Wnt5a and Bmp ligands will further resolve the molecular mechanisms involved in the induction of specific enterocyte villus tip genes by VTTs.

VTTs are ideally suited to orchestrate gene expression programs along the intestinal villi. Their close proximity to the epithelial sheet and their intricate extensions towards the lamina propria facilitate close interactions with both epithelial and other stromal cell types. Unlike the short-lived epithelial cells and the often mobile immune cells, VTTs are static, quiescent and longlived, and can therefore integrate long-term information related to diets and pathologies. The intestine exhibits a remarkable ability to adapt to such perturbations ${ }^{36,37}$. Future work will resolve the roles of VTTs in shaping villus epithelial programs in such perturbed states. The re-appearance of VTTs 3 weeks following their ablation (Fig. 7) supports their importance for preserving the normal epithelial function. It will be important to identify the cellular origins of these re-appearing VTTs. Our study exposed VTTs as major regulators of the expression of villus tip enterocyte genes (Fig. 6e), yet enterocyte gene expression changes throughout the villus axis ${ }^{4}$. It will be interesting to explore the additional effects of zonated luminal signals and potential enterocyte 'internal clocks' in shaping enterocyte zonation. This could be achieved by modulating diets and microbial composition, or by modulating enterocyte turnover, and consequently migration rates, respectively.

Lgr5+ fibroblasts have previously been identified in the lung ${ }^{38}$, suggesting that Lgr5+ stromal cells may constitute a localized mesenchymal niche component in other tissues. It will be important to use similar spatial transcriptomics approaches to explore the cross-talk between the mesenchymal cell populations and zonated immune cell populations ${ }^{26}$, as well as other stromal components such as enteric neurons ${ }^{27}$. Our finding of positive expression of the Lgr5-EGFP-IRES-creERT2 knock-in, as well as the Lgr5-GFP-DTR knock-in cassettes at the villus tip is important for interpreting physiological effects of perturbations driven by the Lgr5 promoter, such as lineage tracing, cell ablation and conditional knock-outs.

Recent efforts for establishing tissue cell atlases rely on the ability to deconvolve the complex spatial interactions of distinct parenchymal and non-parenchymal cell types in each tissue ${ }^{13}$. Our study performs such combined analysis in the mouse small intestine, a prototypical zonated tissue. Similar approaches could be applied to reveal zonated interactions between stroma and epithelial cells in other structured organs.

\section{Methods}

Mice and tissues. All animal studies were approved by the Institutional Animal Care and Use Committee of WIS and UCSF. C57bl6 male mice age 8-10 weeks were obtained from the Harlan laboratories, all mice were fed regular chow ad libitum. All experiments were performed on the same region of the Jejunum, measured as $8 \mathrm{~cm}$ from the stomach-Duodenum boundary (Lgr5+ VTTs appear throughout the small intestine, data not shown). Mice were sacrificed by cervical dislocation. For LCM, jejunum tissues were harvested and embedded directly in OCT without fixation. For smFISH, jejunum tissues were harvested and fixed in $4 \%$ formaldehyde for $3 \mathrm{~h}$, incubated overnight with $30 \%$ sucrose in $4 \%$ formaldehyde and then embedded in OCT in the form of swiss-rolls. Seven micrometer cryosections were used for hybridization. Mouse jejunum cells for single-cell RNAseq were extracted from eight mice. All smFISH quantifications were performed on at least 2 mice. Images in Fig. 3c, d were taken from jejunum tissues of Lgr5-EGFPIRES-CreERT2 mice ${ }^{2}$. Lgr5 $5^{\text {DTRGFP }}$ (Lgr5-GFP-DTR) mice ${ }^{25}$ were housed in the University of California San Francisco (UCSF) animal facility in compliance with all ethical guidelines established by the Institutional Animal Care and Use Committee (IACUC) and Laboratory Animal Resource Center.

Antibodies used in this study. The following antibodies were used for FACS cell isolation: CD31 (PE-Cy7 102418, Biolegend), CD45 (APC-Cy7 103116, PE-Cy7 103114, Biolegend), PDGFRa (Alexa 488 FAB1062G, R\&D systems, APC 135908, Biolegend), EPCAM (PE 118205, Biolegend). All FACS antibodies were used at 1:100 dilution in the presence of TruStain fcX blocker (101320, Biolegend, 1:50). For PDGFRa immunofluorescence staining, goat anti mouse PDGFRa was used as first antibody (AF1062 R\&D systems) at a concentration of $8 \mu \mathrm{g} / \mu \mathrm{l}$ in the smFISH hybridization buffer, Alexa fluor 488 conjugated Donkey anti goat (705-545-147 Jackson laboratories, 1:400) was used as secondary antibody. For E-CADHERIN immunofluorescence staining FITC anti E-CADHERIN (612131 BD Biosciences) was used at dilution of 1:100. 
a

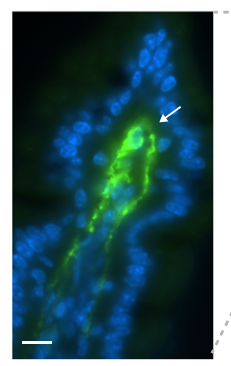

b

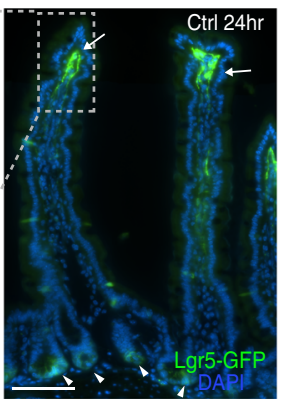

d

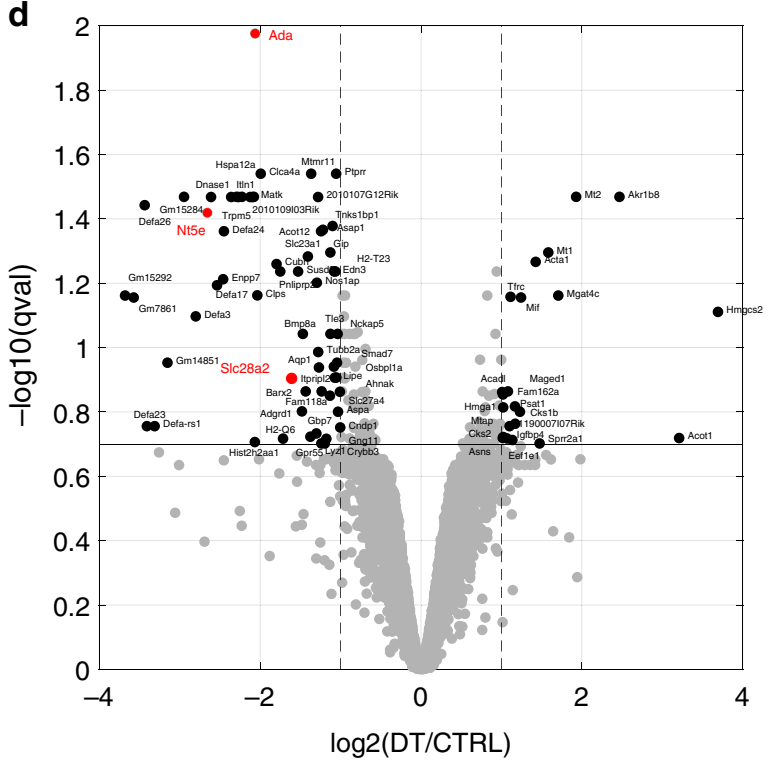

$\mathbf{f}$
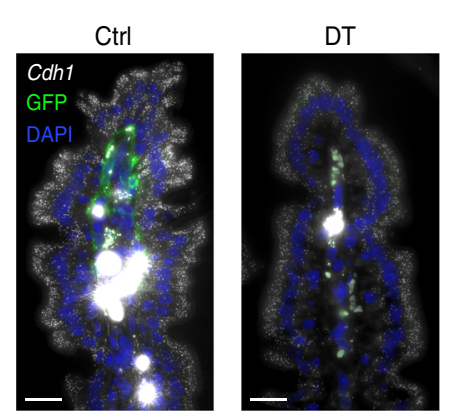

g

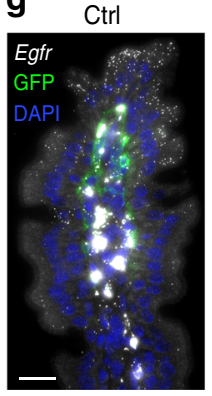

i
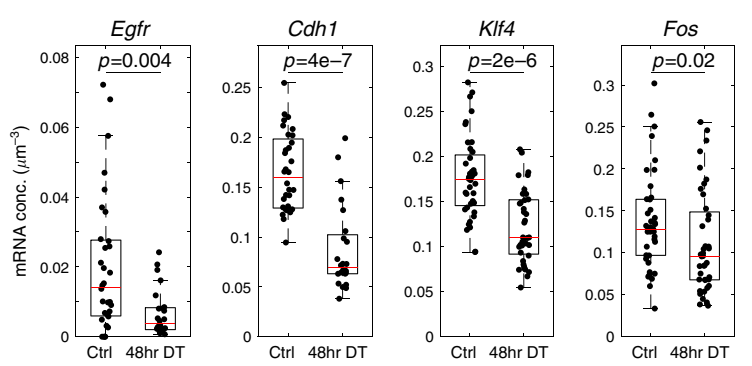

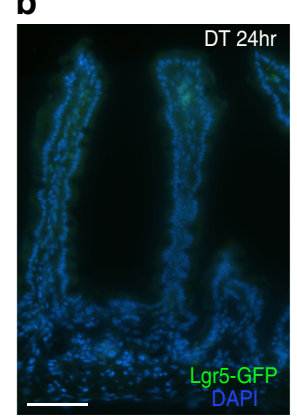

C
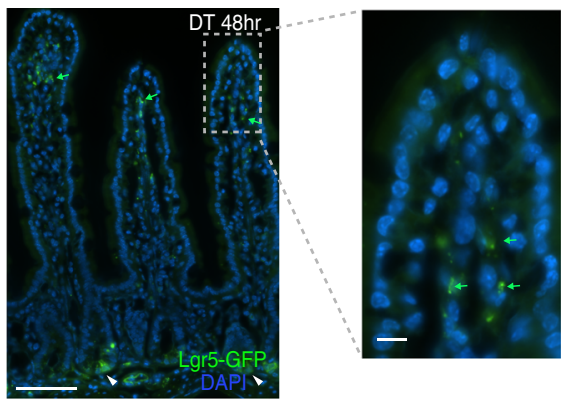

e

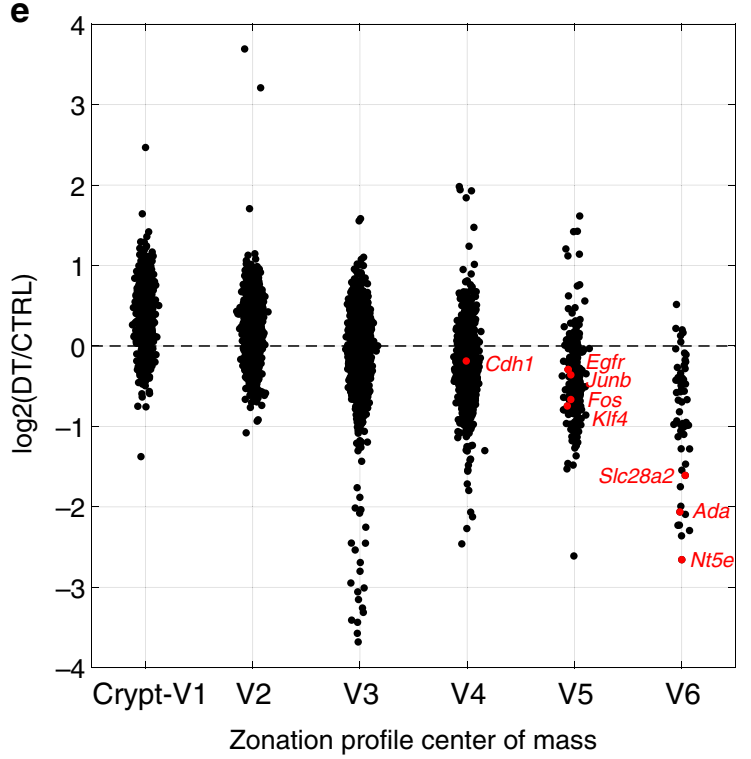

h
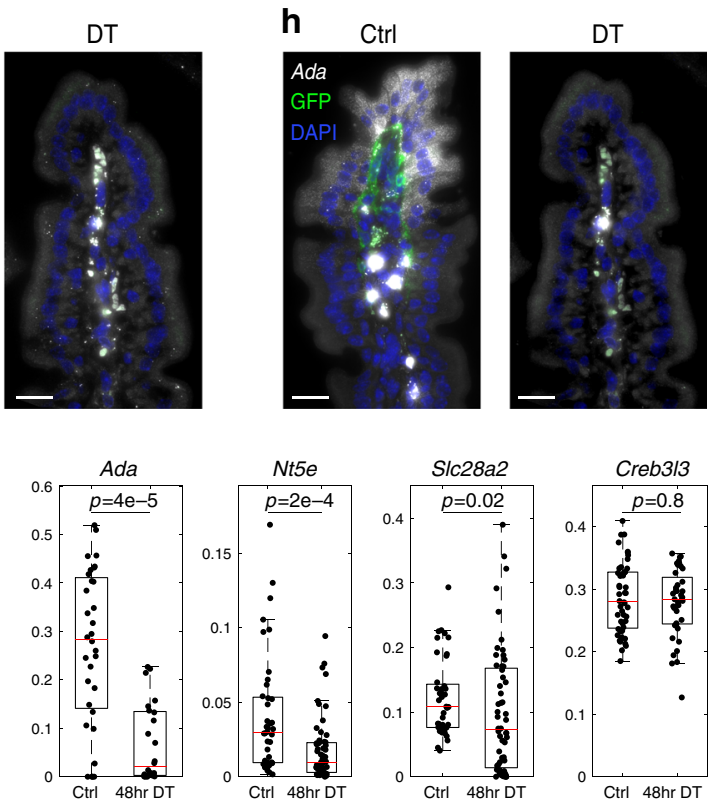

Laser capture microdissection (LCM). Tissue blocks for laser-capture microdissection were freshly prepared at the morning of the collection day. The jejunum part was transected on top of Wattman paper soaked in PBS to support the opened structure in petri dish on ice. The tissue was washed with cold PBS and embedded in OCT on dry ice. LCM protocol was applied as previously described ${ }^{4,39}$ with minor modifications. Sections of $9 \mu \mathrm{m}$ thickness were cut from the frozen block, mounted on polyethylene-naphthalate membrane-coated 518 glass slides (Zeiss, 415190-9081-000), air-dried for $20 \mathrm{~s}$ at room temperature, washed in $70 \%$ ethanol for $25 \mathrm{~s}$, incubated in water for $25 \mathrm{~s}$ (Sigma-Aldrich, W4502), stained with
HistoGene Staining Solution for $20 \mathrm{~s}$ (ThermoFisher Scientific, KIT0401), washed again in water for $25 \mathrm{~s}$. The stained sections were dehydrated with subsequent $25 \mathrm{~s}$ incubations in $70 \%, 95 \%$, and $100 \% \mathrm{EtOH}$ and air-dried for $60 \mathrm{~s}$ before microdissection. Tissue sections were examined using a bright field imaging microscope (Observer.Z1, Zeiss), and microdissected with a UV laser-based unit (PALMMicrobeam, Zeiss). To ensure minimal damage to the surrounding cells, laser intensity and focus were calibrated before each session using Zeiss calibration wizard supplemented with the LCM operating software (Zeiss). Manual detection of analyzed regions in each tested slide and labeling of the desired areas were done 
Fig. 6 VTT ablation perturbs enterocyte expression at the villus tip. a GFP fluorescence (green) observed in both crypt base columnar cells (CBCs, white arrowheads) and VTTs in Lgr5-GFP-DTR mice. Inset on left shows a blow up of villus tip with GFP + VTTs (white arrow). b Both VTTs and CBCs are ablated $24 \mathrm{~h}$ after DT administration, as evident by the lack of GFP fluorescence. c GFP fluorescence re-appears in the crypt (white arrowheads) but not at the villus tip stroma $48 \mathrm{~h}$ after DT administration, indicating stable loss of VTTs. Inset on right shows a blow up of villus tip with no GFP+ VTTs. Green blobs are autofluorescent elements, also marked by green arrows. Scale bar in a-c $-100 \mu \mathrm{m}$, insets scale bar-10 $\mu \mathrm{m}$. d Volcano plot demonstrating the changes in enterocyte gene expression $48 \mathrm{~h}$ following VTT ablation. Enterocyte villus tip genes that are reduced include Ada, Nt5e, and Slc28a2 (red), composing the purine metabolism immune-modullatory tip module ${ }^{4}$. Black genes have q-values lower than 0.2 and max expression higher than $5 \times 10^{-6}$ (Methods). e Enterocyte genes normally induced at the villus tip are reduced in expression $48 \mathrm{~h}$ following VTT ablation. Correlation between change in expression and expression zone $-R=-0.53, p<10^{-60}$. Enterocytes were classified into six villus zones as in Moor et al. ${ }^{4}$. $\mathbf{f}-\mathbf{h}$ smFISH validations of enterocyte villus tip genes that are changed $48 \mathrm{~h}$ following VTT ablation-in each panel Ctrl-left, DT-right. $\mathbf{f}$ Cdh1. $\mathbf{g}$ Egfr. h Ada. Scale bar in $\mathbf{f}-\mathbf{h}-20 \mu \mathrm{m}$. $\mathbf{i}$ Quantification of smFISH experiments, demonstrating that key epithelial villus tip genes are repressed $48 \mathrm{~h}$ after VTT ablation (Egfr, Cdh1, KIf4, Fos, Ada, Nt5e, SIc28a2 $p$-values are indicated), whereas others remain unchanged (Creb3|3), $p$-values were calculated by two-sided Wilcoxon rank sum test. $n=30$ cells examined over 2 mice. Boxes show 25-75 percentiles of the smFISH expression, horizontal red lines are medians, whiskers extend to the most extreme data point within $1.5 \times$ the interquartile range (IQR) from the box.

with PALM $\times 10$ and $\times 20$ lenses. Tissue fragments were catapulted and collected in $0.2 \mathrm{ml}$ adhesive cap tubes (Zeiss, 415190-9191-000). Each capture section was visually confirmed by focusing the PALM on the targeted adhesive cap follow ing the collection session and immediately resuspend in $10.5 \mu \mathrm{l}$ of $1 \times$ reaction buffer from SMART-Seq v4 Ultra Low Input RNA Kit (Clontech, 634888). Tissue lysis was achieved by incubation for $5 \mathrm{~min}$ at room temperature and stored at $-20^{\circ} \mathrm{C}$. Collected samples were stored at $-80^{\circ} \mathrm{C}$ until the library preparation. Three distinct zones spanning approximately equal heights from the crypt-villus border to the villus tip, were collected from long villi (about $500 \mu \mathrm{m}$ )-villus bottom, villus center and villus top (Fig. 1). An area of $\sim 30,000 \mu \mathrm{m}^{2}$ per zone was collected for each mouse by pooling $9-11$ distinct villi from a single tissue section. For mice 1-3 (Supplementary data 1 ) we additionally catapulted crypt stroma and extracted material from two sequential tissue sections for each zone, with the exception of the villus center zone of mouse 3, where one section failed to amplify (Supplementary data 1 , TPM value tab).

SMART-Seq for bulk LCM samples. RNA libraries from the bulk tissues were prepared using SMART-Seq v4 Ultra Low Input RNA Kit (Clontech, 634888), with a 15 PCR cycles for the amplification step. Subsequent steps were applied as mentioned in the protocol. Nextera XT DNA Library Prep Kit (Illumina) was use to finalize the libraries. Library concentration and quality control were determined using NEBNext Library Quant Kit (New England Biolabs) and Agilent High Sensitivity D1000 ScreenTape System (Agilent, 5067-5584). Library final concentration of $2.4 \mathrm{pM}$ was loaded on NextSeq550 (Illumina) sequencing machine aiming for $20 \mathrm{M}$ reads per sample with the following cycle distribution: $38 \mathrm{bp}$ read1, 8 bp index 1,8 bp index 2,38 bp read2.

Stroma LCM bulk RNA-seq analysis. Illumina raw files were converted to FASTQ files using bcl2fastq 2.17 (Illumina), following by pseudoalignment with Kallisto $0.43 .0^{40}$ to a transcriptome index of the GRCm38.94 (Ensembl), filtered to transcripts with "coding_genes" entry. The following flag was used for kallisto: --b 100 (the number of bootstrap samples), --rf-stranded (strand specific reads, first read reverse). Sleuth $0.30 .0^{41}$ running on R 3.5.1 was utilized to create a TPM table (Transcripts Per Million) for each sample (Supplementary data 1).

Stroma LCM data processing. To exclude transcripts that might have originated from residual enterocytes included in the laser microdissected tissue, we filtered out genes that were highly expressed in enterocytes. To this end, we considered the maximal expression of all genes in enterocytes over the zones as measured in Moor et al. ${ }^{4}$. We sorterd these genes by expression levels and removed 31 non-ribosomal and non-mitochondrial genes that were included within the list of highly expressed genes, the cumulative summed expression of which exceeded $50 \%$ of the total enterocyte expression. We normalized the TPM values upon removing these genes by the sum of all remaining TPM. We next divided the gene expression values in each sample by the sum for all genes that individually make up less than $1 \%$ of the sample's summed expression values. For each of the four zones, we computed the means and standard errors of the means over the different samples. Ligand receptor pairs were extracted from Ramilowsky et al. ${ }^{12}$, Matrisome components were extracted from Naba et al. ${ }^{17}$. Only genes with maximal zonation value larger than $5^{*} 10^{-6}$ were considered.

Analysis of II18r1 stromal cells. We used the single cell RNAseq data of Biton et al. ${ }^{26}$ (6558 cells annotated as WT control, Supplementary Fig. 1c). Il18r1 positive cells belonged to a cluster annotated as ' $\mathrm{CD} 4$ '. We performed differential gene expression using Wilcoxon rank-sum tests between the Il18r1+ and Ill18r - cells in that cluster (Supplementary Fig. 1d). We used Immgen My Geneset tab (https:// www.immgen.org) to annotate the 200 genes that had the highest fold-change between Il18r1+ cells and Il18r1 - cells within the CD4-annotated cluster (only genes with expression above $5 \times 10^{-5}$ were used for this analysis). The gene set was enriched in innate lymphoid cells type 3 .

Hybridization and imaging. Probe libraries were designed using the Stellaris FISH Probe Designer Software (Biosearch Technologies, Inc., Petaluma, CA). $7 \mu \mathrm{m}$ thick sections of fixed Jejunum were sectioned onto poly L-lysine coated coverslips and used for smFISH staining. The intestinal sections were hybridized with smFISH probe sets according to a previously published protocol ${ }^{15}$. Briefly, tissues were treated for $10 \mathrm{~min}$ with proteinase $\mathrm{K}(10 \mu \mathrm{g} / \mathrm{ml}$ Ambion AM2546) and washed twice with $2 \times$ SSC (Ambion AM9765). Tissues were incubated in wash buffer $(20 \%$ Formamide Ambion AM9342, $2 \times$ SSC) for 5 min and mounted with the hybridization buffer (10\% Dextran sulfate Sigma D8906, 20\% Formamide, $1 \mathrm{mg} / \mathrm{ml}$ E.coli tRNA Sigma R1753, $2 \times$ SSC, $0.02 \%$ BSA Ambion AM2616, 2 mM Vanadylribonucleoside complex NEB S1402S) mixed with 1:3000 dialution of probes. Hybridization mix was incubated with tissues for overnight in a $30^{\circ} \mathrm{C}$ incubator. SmFISH probe libraries (Supplementary data 7) were coupled to Cy5, TMR or Alexa594. After the hybridization, tissues were washed with wash buffer containing $50 \mathrm{ng} / \mathrm{ml}$ DAPI for $30 \mathrm{~min}$ at $30^{\circ} \mathrm{C}$. Telocytes cells were detected by PDGFR $\alpha$ primary antibody that was added to the smFISH hybridization buffer and Alexa fluor 488 conjugated Donkey anti goat as secondary antibody in GLOX buffer for 20 minutes after DAPI (Sigma-Aldrich, D9542) nuclear staining. All images were taken as scans extending from villus tip to crypt bottom using $\times 100$ magnifications, hence several fields of view were stitched together to cover the whole crypt-villus unit. Stitching was performed with the fusion mode linear blending and default settings of the pairwise stitching plugin ${ }^{42}$ in $\mathrm{Fiji}^{43}$. Quantification of smFISH was done using Image $\mathrm{M}^{44}$. For smFISH quantifications, results were based on at least 30 cells from each region and from at least 2 mice. Dots were counted in the first 3 $\mu \mathrm{m}$ of the Z-stack, and divided by the segmented cell volume to obtain the mRNA concentration per cell. Two-sided Wilcoxon rank-sum tests were used to assess significance. Quantifications of the proportions of Lgr5+ VTTs positive for the EGFP knock-in were done on jejunum tissues of Lgr5-EGFP-Ires-CreERT2 mice ${ }^{2}$

Statistics and reproducibility. All smFISH micrographs are representative of at least 10 images and two mice per gene or region.

Single cell isolation. Due to the difficulties of isolating intestinal stromal cells we applied several dissociation protocols and surface marker staining to obtain a broad sampling of cells. For all mice, cells were isolated from the jejunum. The jejunum was extracted and rinsed in cold PBS. The tissue was opened longitudinally and sliced into small fragments roughly $2 \mathrm{~cm}$ long and incubated in $10 \mathrm{mM}$ EDTA-PBS on ice for $10 \mathrm{~min}$. The tissue was then moved to warm $5 \mathrm{ml} 10 \mathrm{mM}$ EDTA-PBS containing Liberase TM $(100 \mu \mathrm{g} / \mathrm{mL}$, Sigma $)$ and DNaseI $(2 \mathrm{U} / \mathrm{mL}$, Sigma $)$ and incubated at $37^{\circ} \mathrm{C}$ for $20 \mathrm{~min}$ while shaken vigorously every few minutes. At the end of the incubation time, $5 \mathrm{ml}$ of cold PBS was added to the cell suspension. The supernatant was filtered through a $100 \mu \mathrm{m}$ filter and centrifuged at $300 \times g$ for 5 min. the pellet was resuspended in FACS buffer (2 mM EDTA, 0.5\% BSA in PBS) and stained with the required antibodies for flow cytometry sorting.

Isolation of telocytes was performed as previously decribed in ref. ${ }^{9}$. Briefly, Jejunum were dissected and washed thoroughly with Hank's balanced salt solution (HBSS) and were incubated in $5 \mathrm{mM}$ EDTA in HBSS for $10 \mathrm{~min}$ at $4{ }^{\circ} \mathrm{C}$. Intestinal villi were scraped off using a coverslip and the remaining tissue was cut into small pieces and incubated in $5 \mathrm{mM}$ EDTA and HBSS on ice for $10 \mathrm{~min}$ while pipetting to completely remove the remaining epithelium. After vigorous washes, the remaining mesenchymal fraction was incubated with $6 \mathrm{mg} / \mathrm{ml}$ Dispase II $/ 0.05 \%$ trypsin solution (Sigma-Aldrich, 04942078001) supplemented with $1 \mathrm{U} / \mathrm{ml}$ DNaseI (Sigma) at $37^{\circ} \mathrm{C}$, until the solution became cloudy and the mesenchyme was dissociated $(8 \mathrm{~min})$. At the end of the incubation time, $3 \mathrm{ml}$ of warm FCS were added to the cell suspension to stop the digestion. Supernatant was filtered through a $70-\mu \mathrm{m}$ strainer, centrifuged at $2500 \times g$ for $5 \mathrm{~min}$. the pellet was resuspended in 
a

Ctrl

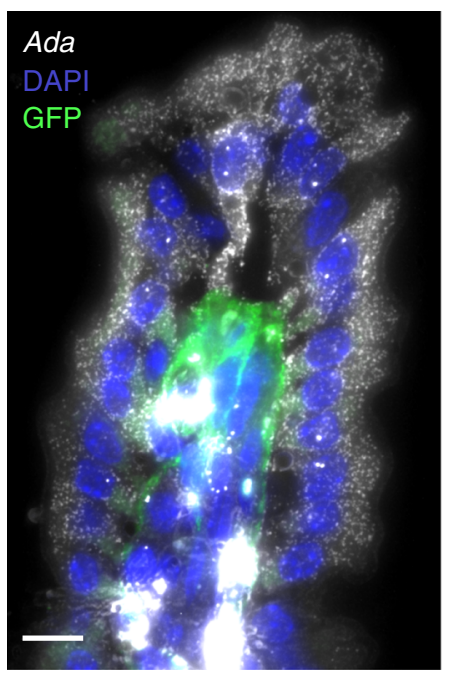

b

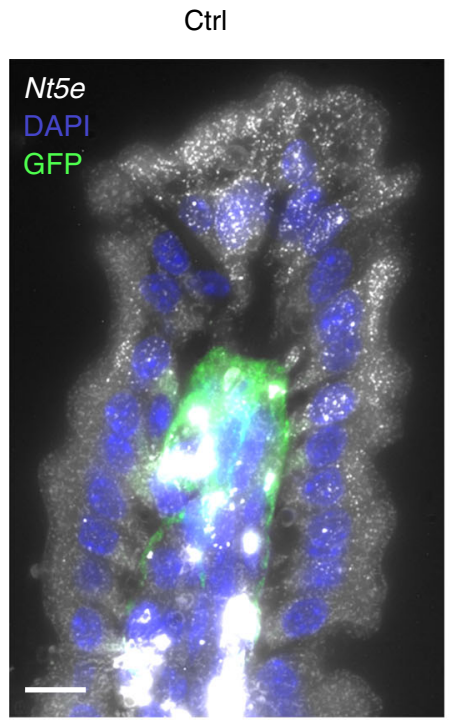

3w DT -

villus without VTTs

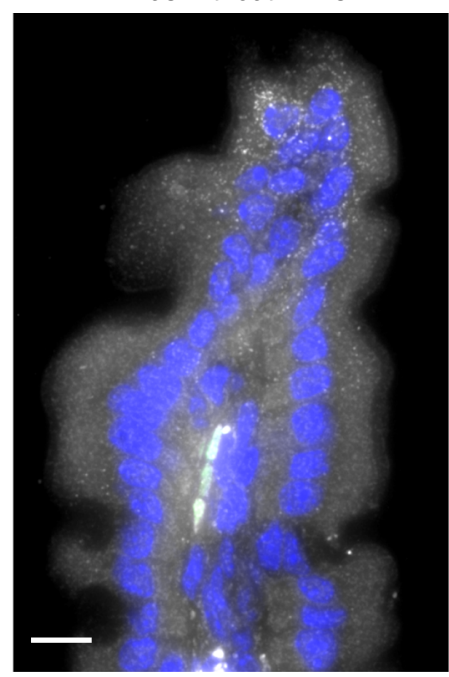

3w DT -

villus without VTTs

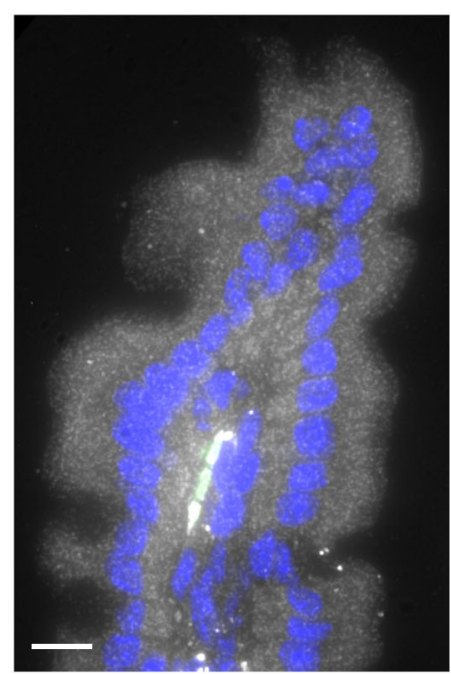

3w DT -

villus with VTTs

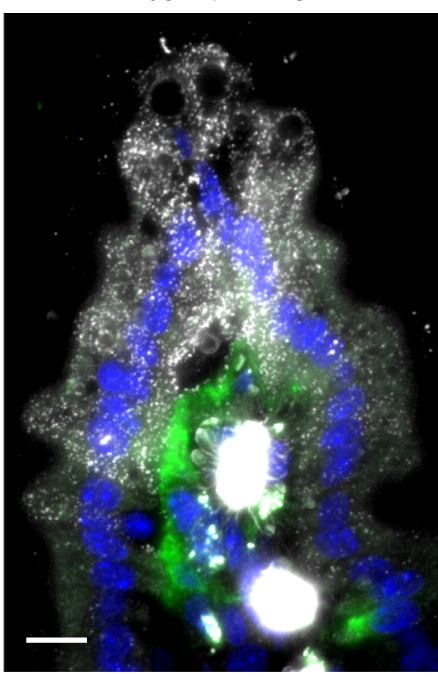

3w DT -

villus with VTTs
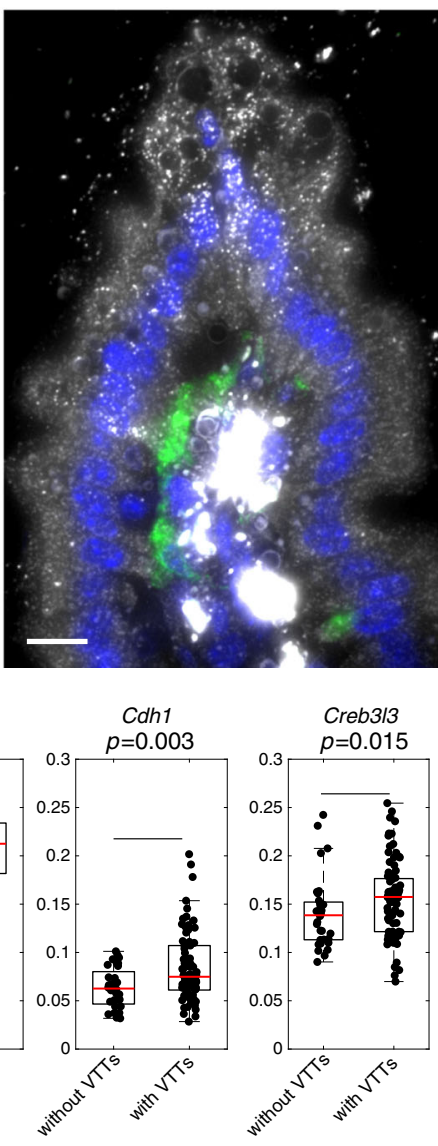

and 30

C
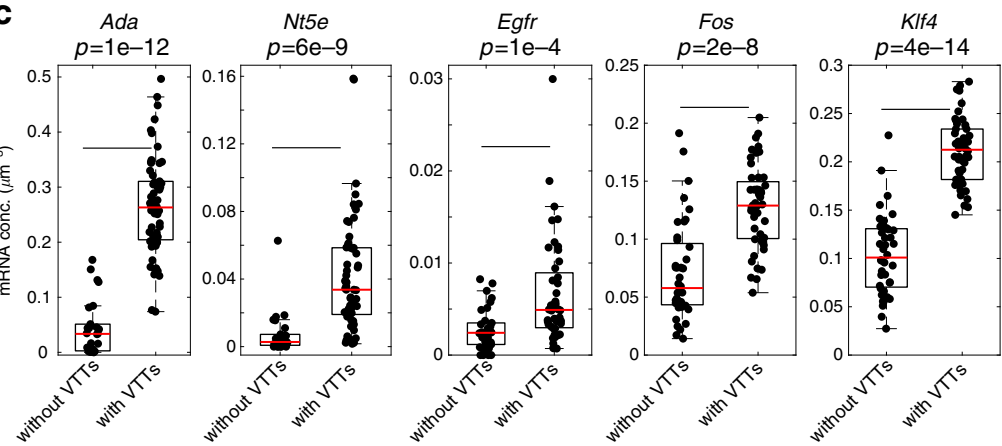

Fig. 7 Analysis of the villus tip epithelial cells of Lgr5-GFP-DTR 3 weeks after VTT ablation shows correlation between the re-appearance of VTTs and the expression of enterocyte tip genes. a smFISH example of Ada showing higher expression in the villus tip with VTTs compared to villus tip without VTTs after 3 weeks. Scale bar $10 \mu \mathrm{m}$. b smFISH example of Nt5e showing higher expression in the villus tip with VTTs compared to villus tip without VTTs after 3 weeks. Scale bar $10 \mu \mathrm{m}$. c Quantification of expression differences between villi with VTTs and villi without VTTs. Measurements were performed over 15 villi per mouse for two mice. Indicated $p$ values were calculated by two-sided Wilcoxon rank sum test. Boxes show 25-75 percentiles of the smFISH expression, horizontal red lines are medians, whiskers extend to the most extreme data point within $1.5 \times$ the interquartile range (IQR) from the box. 
FACS buffer (2 mM EDTA, 0.5\% BSA in PBS) and stained with the required antibodies for flow cytometry sorting.

Single-cell sorting. Single cells were sorted with SORP-FACSAriaII machine (BD Biosciences) using a $100 \mu \mathrm{m}$ nozzle. Dead cells were excluded on the basis of $500 \mathrm{ng} / \mathrm{ml}$ Dapi incorporation. Sorted cells were negative for CD45 and EPCAM and positive for PDGFRa. Cells were sorted into 384 -well cell capture plates containing $2 \mu$ of lysis solution and barcoded poly(T) reverse-transcription (RT) primers for single-cell RNA$\mathrm{seq}^{28}$. Barcoded single cell capture plates were prepared with a Bravo automated liquid handling platform (Agilent) as described previously ${ }^{28}$. Four empty wells were kept in each 384-well plate as a no-cell control during data analysis. Immediately after sorting, each plate was spun down to ensure cell immersion into the lysis solution, snap frozen on dry ice and stored at $-80^{\circ} \mathrm{C}$ until processed.

MARS-Seq library preparation. Single cell libraries were prepared, as described $\mathrm{in}^{28}$. Briefly, mRNA from cells sorted into MARS-Seq capture plates were barcoded and converted into cDNA by reverse transcription reaction and pooled using an automated pipeline. The pooled sample was cleaned using 0.9X SPRI beads and then linearly amplified by T7 in vitro transcription. The resulting RNA was fragmented and converted into sequencing ready library by tagging the samples with pool barcodes and Illumina i7 barcode sequences during ligation, reverse tran scription and PCR. Each pool of cells was tested for library quality and concentration was assessed as described in $^{28}$. Machine raw files were converted to fastq files using bcl2fastq package, to obtain the UMI counts, reads were aligned to the mouse reference genome (GRCm38.84) using zUMI packge ${ }^{45}$ with the following flags that fit the barcode length and the library strandedness: -c 1-7, -m 8-15, -1 66, -B 1, -s 1, -p 16. This analysis resulted in 2217 cells.

scRNAseq data processing. For each single cell and for each gene we first subtracted the estimated background expression. Background was calculated for each 384-well plate separately, as the mean gene expression in the four empty wells. After subtraction, negative values were set to zero. Next, cells with total UMI counts lower than 400 or higher than 8000 and total gene counts lower than 250 were removed. We used Seurat v2.3.4 package in $\mathrm{R}^{29}$ v3.5.3 to visualize and cluster the single cell RNAseq data (Fig. 4, Supplementary Fig. 3). Gene expression measurements (UMIs per gene) were normalized for each cell by the summed UMI, multiplied by a scale factor 10,000, and then log-transformed. To avoid undesired sources of variation in gene expression, we used Seurat to regress out cell-cell variation driven by total number of UMIs, and mitochondrial genes fraction. For detection of variable genes, we set a bottom cutoff of 0.25 and a top cutoff of 4 on the regressed log-transformed average gene expression, as well as a bottom cutoff of 0.5 on the dispersion. Cell clustering was based on PCA dimensionality reduction using the first $11 \mathrm{PCs}$, and a resolution value of 2 .

We used cell type-specific markers to interpret the resulting 3 main clusters, Epcam was highly expressed in the epithelial cells clusters, Ptprc was highly expressed in the immune cells clusters and Pdgfra was highly expressed in the fibroblast cells clusters.

We next focused on the cell clusters that had an average expression of Pdgfra that was higher than $10^{-4}$ per cell (fraction of UMI counts). These included 329 cells. For this new set of cells, we re-ran Seurat with the following parameters; For detection of variable genes, we set a bottom cutoff of 0.25 and a top cutoff of 4 on the regressed log-transformed average gene expression, as well as a bottom cutoff of 1 on the dispersion. Cell clustering was based on PCA dimensionality reduction using the first 5 PCs, and a resolution value of 0.7 . Marker genes were detected with Seurat FindAllMarkers function with parameters min.pct $=0.2$, logfc.threshold $=$ 0.25 (Supplementary data 4).

To identify zonation of the four mesenchymal cell types we examined the summed expression of concise sets of markers that were specific to each cell type. To select these markers, we included genes that had an average expression higher than $5 \times 10^{-6}$ and were expressed in at least 10 cells in the respective cell type. We sorted these genes by the fold change of their expression levels compared to the maximal average expression in the other three mesenchymal cell types. We included the top 20 genes and further excluded genes for which the fold change ratio was lower than 5-fold. Differential gene expression between crypt telocytes and VTTs (Fig. 5a) considered all genes with average expression larger than $10^{-5}$ of cellular UMIs and more than 5 cells positive for the gene in at least one of the two populations. Storey's method was used to compute $q$-values.

Lgr5 + cell ablation. For Lgr5 + cell ablation, male $\operatorname{Lgr} 5^{D T R G F P}$ mice aged 4-5 months were administered $50 \mu \mathrm{g} / \mathrm{kg}$ diphtheria toxin (322326 Sigma) or saline vehicle intraperitoneally. Sample were collected 24 or, $48 \mathrm{~h}$ or 3 weeks post injection. For the $24 \mathrm{~h}$ time points, four DT-injected and two mock-injected mice were used for histological examination (Fig. 6). For the $48 \mathrm{~h}$ time point, four DTinjected and four mock-injected mice were used for histological examination, smFISH and epithelial cell sequencing. In addition, two DT-injected and two mock-injected mice were sacrificed 20 days after administration and used for histological examination and smFISH. For epithelial cell isolation, $3 \mathrm{~cm}$ from the proximal jejunum were extracted and rinsed in cold PBS. The tissue was opened longitudinally and sliced into small fragments roughly $2 \mathrm{~cm}$ long and incubated in
$10 \mathrm{mM}$ EDTA-PBS on ice for $20 \mathrm{~min}$. The tissue was then moved to warm $5 \mathrm{ml}$ $10 \mathrm{mM}$ EDTA-PBS and incubated at $37^{\circ} \mathrm{C}$ for $5 \mathrm{~min}$ while shaken vigorously every few minutes. At the end of the incubation time, $5 \mathrm{ml}$ of cold PBS was added to the cell suspension. The supernatant was filtered through a $100 \mu \mathrm{m}$ filter and centrifuged at $300 \times g$ for $5 \mathrm{~min}$. the pellet was snap frozen in liquid nitrogen and was processed for bulk RNA sequencing. For smFISH blocks, $5 \mathrm{~cm}$ from the distal jejunum was fixed immediately in $4 \% \mathrm{FA}$ and processed as mentioned before.

Bulk RNA sequencing of ablation experiment samples. Snap frozen cells were thawed into TRI reagent (sigma), RNA was isolated by Direct-zol RNA MiniPrep kit (Zymo research) according to the manufacturer instructions. RNA was processed by the mcSCRBseq protocol ${ }^{46}$ with minor modifications. RT reaction was applied on $10 \mathrm{ng}$ of total RNA with a final volume of $10 \mu \mathrm{l}(1 \times$ Maxima H Buffer $1 \mathrm{mM}$ dNTPs, $2 \mu \mathrm{M}$ TSO* E5V6NEXT, 7.5\% PEG8000, 20U Maxima H enzyme, $1 \mu \mathrm{l}$ barcoded RT primer). Subsequent steps were applied as mentioned in the protocol. Library preparation was done using Nextera XT kit (Illumina) on $0.6 \mathrm{ng}$ amplified cDNA. Library final concentration of $2.2 \mathrm{pM}$ was loaded on NextSeq 500 (Illumina) sequencing machine aiming for $20 \mathrm{M}$ reads per sample. Raw files were converted to FASTQ files using bcl2fastq package, to obtain the UMI counts, fast reads were aligned to the mouse reference genome (GRCm38.84) using zUMI packge ${ }^{45}$ with the following parameters RD1 $16 \mathrm{bp}$, RD2 66 bp with a barcode (i7) length of $8 \mathrm{bp}$. UMI counts were processed with the edgeR package ${ }^{47}$. Our analysis included four intestinal samples of DT-injected mice and four from mock-injected, $48 \mathrm{~h}$ after injection (Supplementary data 5). We first filtered the genes by expression to maintain only genes with expression greater than $10^{-5}$ of the summed UMI counts of the sample in at least 2 samples and next ran the calcNormFactors function with default settings. We used the "robust" option in the glmQLFit function to robustly estimate the QL dispersion and glmQLFTest with default parameters to compute differential gene expression with Benjamini-Hochberg correction for multiple hypotheses (Supplementary data 6). Figure 6d shows the volcano plots of all genes with with maximal expression higher than $5 \times 10^{-6}$ in at least one of the samples, as well as maximal zonation higher than $5 \times 10^{-6}$ in Moor et al. ${ }^{4}$.

Reporting summary. Further information on research design is available in the Nature Research Reporting Summary linked to this article.

\section{Data availability}

All data has been deposited in the GEO database with accession code GSE134479.

\section{Code availability}

All codes used in this study are available upon request.

Received: 10 December 2019; Accepted: 24 March 2020; Published online: 22 April 2020

\section{References}

1. Flier, L. G. vander \& Clevers, H. Stem cells, self-renewal, and differentiation in the intestinal epithelium. Annu. Rev. Physiol. 71, 241-260 (2009).

2. Barker, N. et al. Identification of stem cells in small intestine and colon by marker gene Lgr5. Nature 449, 1003-1007 (2007).

3. Potten, C. S. Stem cells in gastrointestinal epithelium: numbers, characteristics and death. Philos. Trans. R. Soc. Lond. B. Biol. Sci. 353, 821-830 (1998).

4. Moor, A. E. et al. Spatial reconstruction of single enterocytes uncovers broad zonation along the intestinal villus axis. Cell https://doi.org/10.1016/j. cell.2018.08.063 (2018).

5. Beumer, J. et al. Enteroendocrine cells switch hormone expression along the crypt-to-villus BMP signalling gradient. Nat. Cell Biol. 20, 909-916 (2018).

6. Gehart, $\mathrm{H}$. et al. Identification of enteroendocrine regulators by real-time single-cell differentiation mapping. Cell 176, 1158-1173.e16 (2019).

7. Roulis, M. \& Flavell, R. A. Fibroblasts and myofibroblasts of the intestinal lamina propria in physiology and disease. Differentiation 92, 116-131 (2016).

8. Degirmenci, B., Valenta, T., Dimitrieva, S., Hausmann, G. \& Basler, K. GLI1expressing mesenchymal cells form the essential Wnt-secreting niche for colon stem cells. Nature 558, 449-453 (2018).

9. Shoshkes-Carmel, M. et al. Subepithelial telocytes are an important source of Wnts that supports intestinal crypts. Nature 557, 242-246 (2018).

10. Kinchen, J. et al. Structural remodeling of the human colonic mesenchyme in inflammatory bowel disease. Cell 175, 372-386.e17 (2018).

11. Aoki, R. et al. Foxl1-expressing mesenchymal cells constitute the intestinal stem cell niche. Cell. Mol. Gastroenterol. Hepatol. 2, 175-188 (2016).

12. Ramilowski, J. A. et al. A draft network of ligand-receptor-mediated multicellular signalling in human. Nat. Commun. 6, 7866 (2015).

13. Vento-Tormo, R. et al. Single-cell reconstruction of the early maternal-fetal interface in humans. Nature 563, 347-353 (2018). 
14. Tan, D. W.-M. \& Barker, N. Chapter three-intestinal stem cells and their defining niche. In Current Topics in Developmental Biology (ed. Rendl, M.) Vol. 107, 77-107 (Academic Press, 2014).

15. Itzkovitz, S. et al. Single molecule transcript counting of stem cell markers in the mouse intestine. Nat. Cell Biol. 14, 106-114 (2011).

16. He, X. C. et al. BMP signaling inhibits intestinal stem cell self-renewal through suppression of Wnt- $\beta$-catenin signaling. Nat. Genet. 36, 1117 (2004).

17. Naba, A. et al. The extracellular matrix: tools and insights for the "omics" era. Matrix Biol. 49, 10-24 (2016).

18. Simon-Assmann, P., Spenle, C., Lefebvre, O. \& Kedinger, M. The role of the basement membrane as a modulator of intestinal epithelial-mesenchymal interactions. Prog. Mol. Biol. Transl. Sci. 96, 175-206 (2010).

19. Meran, L., Baulies, A. \& Li, V. S. W. Intestinal stem cell niche: the extracellular matrix and cellular components. Stem Cells Int. 2017, 15 (2017).

20. Karlsson, L., Lindahl, P., Heath, J. K. \& Betsholtz, C. Abnormal gastrointestinal development in PDGF-A and PDGFR- $\alpha$ deficient mice implicates a novel mesenchymal structure with putative instructive properties in villus morphogenesis. Development 127, 3457-3466 (2000).

21. Cretoiu, D., Cretoiu, S. M., Simionescu, A. A. \& Popescu, L. M. Telocy tes, a distinct type of cell among the stromal cells present in the lamina propria of jejunum. Histol. Histopathol. 27, 1067-1078 (2012).

22. Greicius, G. et al. PDGFRa + pericryptal stromal cells are the critical source of Wnts and RSPO3 for murine intestinal stem cells in vivo. Proc. Natl Acad. Sci. USA 115, E3173-E3181 (2018).

23. Stzepourginski, I. et al. CD34+ mesenchymal cells are a major component of the intestinal stem cells niche at homeostasis and after injury. Proc. Natl Acad. Sci. USA 114, E506-E513 (2017).

24. Carmon, K. S., Gong, X., Lin, Q., Thomas, A. \& Liu, Q. R-spondins function as ligands of the orphan receptors LGR4 and LGR5 to regulate Wnt/beta-catenin signaling. Proc. Natl Acad. Sci. USA 108, 11452-11457 (2011).

25. Tian, H. et al. A reserve stem cell population in small intestine renders Lgr5positive cells dispensable. Nature https://doi.org/10.1038/nature10408 (2011).

26. Biton, $\mathrm{M}$. et al. T helper cell cytokines modulate intestinal stem cell renewal and differentiation. Cell 175, 1307-1320.e22 (2018).

27. Zeisel, A. et al. Molecular architecture of the mouse nervous system. Cell 174, 999-1014.e22 (2018)

28. Jaitin, D. A. et al. Massively parallel single-cell RNA-Seq for marker-free decomposition of tissues into cell types. Science 343, 776-779 (2014).

29. Butler, A., Hoffman, P., Smibert, P., Papalexi, E. \& Satija, R. Integrating singlecell transcriptomic data across different conditions, technologies, and species. Nat. Biotechnol. 36, 411-420 (2018).

30. Horiguchi, H. et al. ANGPTL2 expression in the intestinal stem cell niche controls epithelial regeneration and homeostasis. EMBO J. 36, 409-424 (2017).

31. Wei, X. et al. Spatially restricted stromal Wnt signaling restrains prostate epithelial progenitor growth through direct and indirect mechanisms. Cell Stem Cell 24, 753-768.e6 (2019).

32. Kirsch, N. et al. Angiopoietin-like 4 is a Wnt signaling antagonist that promotes LRP6 turnover. Dev. Cell 43, 71-82.e6 (2017).

33. Tetteh, P. W. et al. Replacement of lost Lgr5-positive stem cells through plasticity of their enterocyte-lineage daughters. Cell Stem Cell https:/doi.org/ 10.1016/j.stem.2016.01.001 (2016).

34. Amin, N. \& Vincan, E. The Wnt signaling pathways and cell adhesion. Front. Biosci. Landmark Ed. 17, 784-804 (2012).

35. Bengoa-Vergniory, N., Gorroño-Etxebarria, I., González-Salazar, I. \& Kypta, R. M. A switch from canonical to noncanonical Wnt signaling mediates early differentiation of human neural stem cells. Stem Cells 32, 3196-3208 (2014).

36. Beyaz, S. et al. High-fat diet enhances stemness and tumorigenicity of intestinal progenitors. Nature 531, 53-58 (2016).

37. Yilmaz, Ö. H. et al. mTORC1 in the Paneth cell niche couples intestinal stemcell function to calorie intake. Nature 486, 490-495 (2012)

38. Lee, J.-H. et al. Anatomically and functionally distinct lung mesenchymal populations marked by Lgr5 and Lgr6. Cell 170, 1149-1163.e12 (2017).

39. Moor, A. E. et al. Global mRNA polarization regulates translation efficiency in the intestinal epithelium. Science 357, 1299-1303 (2017).

40. Bray, N. L., Pimentel, H., Melsted, P. \& Pachter, L. Near-optimal probabilistic RNA-seq quantification. Nat. Biotechnol. 34, 525-527 (2016).

41. Pimentel, H., Bray, N. L., Puente, S., Melsted, P. \& Pachter, L. Differential analysis of RNA-seq incorporating quantification uncertainty. Nat. Methods 14, 687-690 (2017).

42. Preibisch, S., Saalfeld, S. \& Tomancak, P. Globally optimal stitching of tiled 3D microscopic image acquisitions. Bioinforma. Oxf. Engl. 25, 1463-1465 (2009).
43. Schindelin, J. et al. Fiji: an open-source platform for biological-image analysis. Nat. Methods 9, 676-682 (2012).

44. Lyubimova, A. et al. Single-molecule mRNA detection and counting in mammalian tissue. Nat. Protoc. 8, 1743-1758 (2013).

45. Parekh, S., Ziegenhain, C., Vieth, B., Enard, W. \& Hellmann, I. zUMIs-A fast and flexible pipeline to process RNA sequencing data with UMIs. GigaScience 7, giy059 (2018).

46. Bagnoli, J. W. et al. Sensitive and powerful single-cell RNA sequencing using mcSCRB-seq. Nat. Commun. 9, 2937 (2018).

47. Robinson, M. D., McCarthy, D. J. \& Smyth, G. K. edgeR: a Bioconductor package for differential expression analysis of digital gene expression data. Bioinformatics 26, 139-140 (2010).

\section{Acknowledgements}

S.I. is supported by the Henry Chanoch Krenter Institute for Biomedical Imaging and Genomics, The Leir Charitable Foundations, Richard Jakubskind Laboratory of Systems Biology, Cymerman-Jakubskind Prize, The Lord Sieff of Brimpton Memorial Fund, the I-CORE program of the Planning and Budgeting Committee and the Israel Science Foundation (grants 1902/ 12 and 1796/12), the Israel Science Foundation grant No. 1486/16, the Broad Institute-Israel Science Foundation grant No. 2615/18, the Chan Zuckerberg Initiative grant No. CZF2019-002434, the European Research Council under the European Union's Seventh Framework Programme (FP7/2007-2013)/ERC grant agreement number 335122, the Bert L. and N. Kuggie Vallee Foundation and the Howard Hughes Medical Institute (HHMI) international research scholar award. O.D.K. is supported by NIH R35-DE026602 and U01-DK103147.

\section{Author contributions}

S.I., K.B.H., and M.S.C. conceived the study. K.B.H. designed and performed most of the experiments. H.M. and A.E.M. performed LCM experiments. K.B.H., N.W.C., and I.A. performed single cell RNAseq. K.B.H., M.R., L.F., Y.H., and D.R.M. performed smFISH experiments. R.K.Z, D.C.A., K.B.H. performed the the $\operatorname{Lgr} 5^{D T R G F P}$ mice experiments with supervision of O.D.K. and F.J.D.S. S.I. and K.B.H. performed bioinformatics analysis. A.E. and I.A. assisted with data analysis. S.I. and K.B.H. wrote the manuscript. S.I. and M.S.C. supervised the study. All authors discussed the results and commented on the manuscript.

\section{Competing interests}

The authors declare no competing interests.

\section{Additional information}

Supplementary information is available for this paper at https://doi.org/10.1038/s41467 020-15714-x.

Correspondence and requests for materials should be addressed to M.S.-C. or S.I.

Peer review information Nature Communications thanks Prisca Liberali and the other, anonymous, reviewer(s) for their contribution to the peer review of this work.

Reprints and permission information is available at http://www.nature.com/reprints

Publisher's note Springer Nature remains neutral with regard to jurisdictional claims in published maps and institutional affiliations.

Open Access This article is licensed under a Creative Commons Attribution 4.0 International License, which permits use, sharing, adaptation, distribution and reproduction in any medium or format, as long as you give appropriate credit to the original author(s) and the source, provide a link to the Creative Commons license, and indicate if changes were made. The images or other third party material in this article are included in the article's Creative Commons license, unless indicated otherwise in a credit line to the material. If material is not included in the article's Creative Commons license and your intended use is not permitted by statutory regulation or exceeds the permitted use, you will need to obtain permission directly from the copyright holder. To view a copy of this license, visit http://creativecommons.org/ licenses/by/4.0/.

\section{(C) The Author(s) 2020}

